\title{
Rayleigh waves in a non-homogeneous, thermo, magneto, prestressed granular material with variable density under the effect of gravity
}

\author{
Rajneesh Kakar ${ }^{1, *}$, Shikha Kakar ${ }^{2}$ \\ ${ }^{1}$ Principal, DIPS Polytechnic College, Hoshiarpur, Punjab, India \\ ${ }^{2}$ Assistant Professor, SBBSIET Padhiana, Jalandhar, Punjab, India
}

Email address:

rkakar_163@rediffmail.com (R. Kakar)

\section{To cite this article:}

Rajneesh Kakar, Shikha Kakar. Rayleigh Waves in a Non-Homogeneous, Thermo, Magneto, Prestressed Granular Material with Variable Density under the Effect of Gravity. American Journal of Modern Physics. Vol. 2, No. 1, 2013, pp. 7-20.

doi: 10.11648/j.ajmp.20130201.12

\begin{abstract}
The effect of various inhomogeneity factors on propagation of Rayleigh waves in prestressed elastic granular medium are investigated. Inhomogeneities have been assumed to vary exponentially with depth. Lame's potential is used to solve the problem. Some special cases have also been deduced. Dispersion curves are computed numerically and presented graphically by using MathCAD. The results indicate that on neglecting various effects of inhomogeneity, initial stress and gravity, the calculations agrees with classical theories.
\end{abstract}

Keywords: Inhomogeneous Granular Media, Gravity, Initial Stress, Rayleigh Waves

\section{Introduction}

Rayleigh waves are a combination of compression and shear waves. Their traveling speed is slightly smaller than bulk shear waves [1]. The propagation of Rayleigh waves in a granular media is of considerable importance in soil mechanics, geophysics and earthquake science etc.

Lot of literature on Rayleigh waves in granular media is available in articles written by many authors such as Datta [2], Abd-Alla, El-Naggar and Ahmed [3, 4, 5, 10], Sharma JN and Kaur [6], Oshima [7-8], Paria [9]. Rayleigh waves in a magneto-elastic material under the influence of initial stress and a gravity field were discussed by Abd-Alla et al. [11]. Willson W and Yu CP and Tang S [12, 13] investigated the problem of the propagation of magneto-thermo, elastic plane waves. Gupta et al. [14] investigated surface waves in non homogeneous granular material under gravity.

Recently, Xianhai Song et al. [15] studied the Application of particle swarm optimization to interpret Rayleigh dispersion curves. Kakar [16, 17, 18, and 19] has discussed Rayleigh waves in non-homogeneous granular media, viscoelastic and in elastic media. Some problems on Love waves propagating in piezoelectric material under the effect of an electro-elastic field were also discussed by Britan [20], Danoyan [21], Du et al. [22], Eskandari [23] and Du et al.
[24].

However, the combined effect of temperature and magnetic field on Rayleigh waves propagating in nonhomogeneous granular media has not been discussed so far; therefore authors solved the problem of Rayleigh waves propagating in a non- homogeneous granular medium under various inhomogeneities and the results presented in this paper should prove useful for researchers in material science.

In this paper, the influence of magnetic field, gravity, temperature, initial compression and non- homogeneity on the propagation of Rayleigh waves in a granular half space supporting a different granular layer is studied. The frequency equation of Rayleigh waves is obtained in the form of a 9x9 determinant. The non-homogeneities are assumed to vary exponentially with depth. It is assumed that the, medium is discontinuous and it is made up of numerous large or small grains. These grains not only translate but also rotate about its centre of gravity as shown in fig. 1 .

The motion of these grains produce friction, therefore the concept of friction has taken in the governing equations. Also, when the gravity, temperature, magnetic field, initial compression and non-homogeneity are neglected, the frequency equation is in well agreement with the corresponding classical result. The results are explained graphically by choosing standard parameters of the medium. 


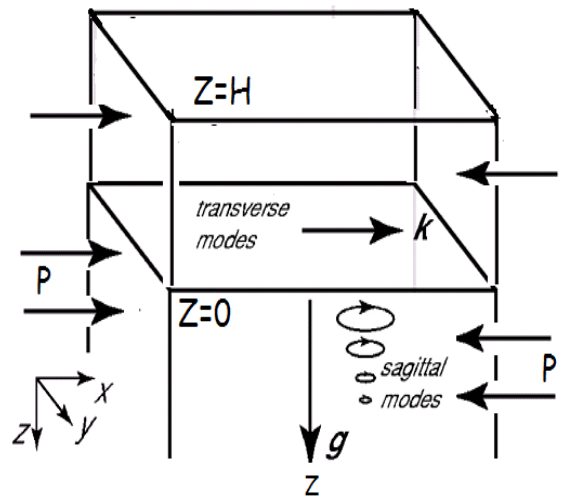

Figure 1. Schematic of the problem.

\section{Formulation of the Problem}

We consider Oxyz Cartesian co-ordinate system with o being any point on the free surface, here we consider the free surface and interface of granular layer resting on nonhomogeneous granular half space bounded by two planes of different material given by $\mathrm{z}=0$ and $\mathrm{z}=\mathrm{H}$ respectively. Also it is assumed that oz being normal to half space and Rayleigh wave propagation in the positive direction of $x$ axis.

Here it is also assumed that at a great distance from centre of disturbance, the wave propagation is two dimensional and is polarized in xz-plane.

Therefore the displacement components along $\mathrm{x}$ and $\mathrm{z}$ directions are non-zero i.e. $\mathrm{u}_{1}$ and $\mathrm{u}_{3}$ are non-zero while $\mathrm{u}_{2}$ is zero. Also, it is assumed that, wave is surface wave as the disturbance is extensively confined to the boundary. $(\xi, \eta, \zeta)$ gives the rotation vector of the grain about its centre of gravity so there exists a stress tensor and stress couple which are non-symmetric i.e. $\sigma_{\mathrm{ij}} \neq \sigma_{\mathrm{ji}}$ and $\mathrm{M}_{\mathrm{ij}} \neq \mathrm{M}_{\mathrm{ij}}$.

The stress tensor $\sigma_{\mathrm{ij}}$ can be expressed in symmetric and anti-symmetric tensors $\tau_{\mathrm{ij}}$ and $\tau_{\mathrm{ij}}$

i.e.

$$
\sigma_{\mathrm{ij}}=\tau_{\mathrm{ij}}+\tau^{\prime} \mathrm{ij},
$$

where

$$
\tau_{\mathrm{ij}}=\frac{1}{2}\left(\sigma_{\mathrm{ij}}+\sigma_{\mathrm{ji}}\right)
$$

and

$$
\tau_{\mathrm{ij}}^{\prime}=\frac{1}{2}\left(\sigma_{\mathrm{ij}}-\sigma_{\mathrm{ji}}\right)
$$

Further symmetric strain tensor are given by relation

$$
\mathrm{e}_{\mathrm{ij}}=\mathrm{e}_{\mathrm{ji}}=\frac{1}{2}\left(\frac{\partial u_{i}}{\partial x_{j}}+\frac{\partial u_{j}}{\partial x_{i}}\right) .
$$

The anti-symmetric stresses $\tau_{i j}^{\prime}$ are given by

$$
\begin{gathered}
\tau_{23}^{\prime}=-F \frac{\partial \xi}{\partial t}, \tau_{31}^{\prime}=-F \frac{\partial \eta}{\partial t}, \tau_{12}^{\prime}=-F \frac{\partial \mathrm{z}}{\partial t}, \\
\tau_{11}^{\prime}=\tau_{22}^{\prime}=\tau_{33}^{\prime}=0,
\end{gathered}
$$

where $\mathrm{F}$ be the co-efficient of friction.

The stress couple $\mathrm{M}_{\mathrm{ij}}$ is given by

$$
\mathrm{M}_{\mathrm{ij}}=M v_{\mathrm{ij}},
$$

where $\mathrm{M}$ be the elastic constant,

$$
\begin{gathered}
v_{11}=\frac{\partial \xi}{\partial x}, v_{22}=0, v_{33}=\frac{\partial \xi}{\partial z} ; v_{23}=0, v_{31}=\frac{\partial \xi}{\partial z} \\
v_{12}=\frac{\partial}{\partial x}\left(\mathrm{w}_{2}+\eta\right), v_{32}=\frac{\partial}{\partial z}\left(\mathrm{w}_{2}+\eta\right), \\
v_{13}=\frac{\partial \mathrm{z}}{\partial x}, v_{21}=0
\end{gathered}
$$

where rotation vector

$$
\begin{gathered}
\mathrm{w}_{1}=\frac{1}{2}\left(\mathrm{u}_{3, \mathrm{y}}-\mathrm{u}_{2, \mathrm{z}}\right), \\
\mathrm{w}_{2}=\frac{1}{2}\left(\mathrm{u}_{1, \mathrm{z}}-\mathrm{u}_{3, \mathrm{x}}\right), \mathrm{w}_{3}=\frac{1}{2}\left(\mathrm{u}_{2, \mathrm{x}}-\mathrm{u}_{1, \mathrm{y}}\right) .
\end{gathered}
$$

Let $g$ be the acceleration due to gravity and $\rho$ be the density of the material,

The state of initial stresses are given by

$$
\left.\begin{array}{rl} 
& =\sigma ; i=j \\
\sigma_{\mathrm{ij}} & =0 ; i \neq j
\end{array}\right\} \text { where } \mathrm{i}, \mathrm{j}=1,2,3
$$

Further, $\sigma$ is a function of $\mathrm{z}$.

The Eq. of equilibrium of initial stresses are

$$
\sigma_{, \mathrm{x}}=0 ; \sigma_{\mathrm{z}}-\rho \mathrm{g}=0
$$

The problem is dealing with magnetoelasticity. Therefore the basic equations will be electromagnetism and elasticity. Let us consider that the medium is a perfect electric conductor, we take the linearized Maxwell equations governing the electromagnetic field, taking into account absence of the displacement current (in system-international unit) in the form

$$
\vec{\nabla} \cdot \overrightarrow{\mathrm{E}}=0, \vec{\nabla} \cdot \overrightarrow{\mathrm{B}}=0, \vec{\nabla} \times \overrightarrow{\mathrm{E}}=-\frac{\overrightarrow{\partial \mathrm{B}}}{\partial t},
$$




$$
\vec{\nabla} \times \overrightarrow{\mathrm{B}}=\mu_{e} \varepsilon_{e} \frac{\overrightarrow{\partial \mathrm{E}}}{\partial t} .
$$

Where, $\overrightarrow{\mathrm{E}}, \overrightarrow{\mathrm{B}}, \mu_{e}$ and $\varepsilon_{e}$ are electric field, magnetic field induction, permeability and permittivity of the medium.

The value of magnetic field intensity is

$$
\overrightarrow{\mathrm{H}}(0,0, \mathrm{H})=\overrightarrow{\mathrm{H}}_{0}+\overrightarrow{\mathrm{H}}_{i}
$$

We have considered an elastic solid under constant primary magnetic field $\overrightarrow{\mathrm{H}}_{0}$ acting on the y-axis, gravity $\mathrm{g}$, perturbation $\overrightarrow{\mathrm{H}}_{i}$ and an initial stress $\mathrm{P}$ along the $\mathrm{x}$-axis.

It is assumed that prior to the existence of any disturbance both the media are everywhere at the constant absolute temperature $\mathrm{T}_{0}$. $\mathrm{T}$ is the absolute temperature over the initial temperature $\mathrm{T}_{0}$. In a thermo viscoelastic solid, the thermal parameters $\Xi$ are given by $\Xi=(3 \lambda+2 \mu) \alpha_{t}$, where $\alpha_{t}$ be the coefficient of linear expansion of solid.

The dynamical equations of motion in the $\mathrm{x}$ and $\mathrm{z}$ dimensions of granular medium under gravity are

$$
\begin{gathered}
\sigma_{11, \mathrm{x}}+\sigma_{13, \mathrm{z}}-\rho \mathrm{gu}_{3, \mathrm{x}}+P\left(\omega_{3, y}-\omega_{2, \mathrm{z}}\right)+_{\tau_{31, \mathrm{z}}^{\prime}}=\rho \mathrm{u}_{1, \mathrm{tt}}, \\
\sigma_{12, \mathrm{x}}+\sigma_{23, \mathrm{z}}+\tau_{12, \mathrm{x}}^{\prime}+P \omega_{3, y}-\tau_{23, \mathrm{z}}^{\prime}=0 \\
\sigma_{13, \mathrm{x}}+\sigma_{33, \mathrm{z}}+\rho g \mathrm{gu}_{1, \mathrm{x}}-P \omega_{2, x}-\tau_{31, \mathrm{x}}^{\prime}=\rho \mathrm{u}_{3, \mathrm{t}},
\end{gathered}
$$

and

$$
\begin{gathered}
\tau_{23}^{\prime}+\sigma_{23}-\sigma_{32}+M_{11, \mathrm{x}}+M_{31, \mathrm{z}}=0, \\
\tau_{31}^{\prime}+\sigma_{31}-\sigma_{13}+M_{12, \mathrm{x}}+M_{32, \mathrm{z}}=0, \\
\tau_{12}^{\prime}+\sigma_{12}-\sigma_{21}+M_{13, \mathrm{x}}+M_{33, \mathrm{z}}=0 .
\end{gathered}
$$

\section{Solution of Problem}

Further the stress components are given by

$$
\begin{array}{r}
\sigma_{11}=(\lambda+2 \mu) \mathrm{u}_{1, \mathrm{x}}+\lambda \mathrm{u}_{3, \mathrm{z}}+\Delta \mu_{e} H_{0}^{2}-\Xi T \\
=\mathrm{C}_{11} \mathrm{u}_{1, \mathrm{x}}+\mathrm{C}_{13} \mathrm{u}_{3, \mathrm{z}}+\Delta \mu_{e} H_{0}^{2}-\Xi T, \\
\sigma_{33}=\lambda \mathrm{u}_{1, \mathrm{x}}+(\lambda+2 \mu) \mathrm{u}_{3, \mathrm{z}}+\Delta \mu_{e} H_{0}^{2}-\Xi T \\
=\mathrm{C}_{13} \mathrm{u}_{1, \mathrm{x}}+\mathrm{C}_{33} \mathrm{u}_{3, \mathrm{z}}+\Delta \mu_{e} H_{0}^{2}-\Xi T, \\
\sigma_{13}=\mu\left(\mathrm{u}_{3, \mathrm{x}}+\mathrm{u}_{1, \mathrm{z}}\right)=\mathrm{C}_{44}\left(\mathrm{u}_{3, \mathrm{x}}+\mathrm{u}_{1, \mathrm{z}}\right) .
\end{array}
$$

putting Eq. (10), Eq. (5), Eq. (6) and Eq. (4) in Eq. (9) ; we get

$$
\begin{aligned}
& (\lambda+2 \mu+P) u_{1, x x}+\mu u_{1, z z}+(\lambda+\mu+P) u_{3, x z}+u_{1, x} \frac{\partial}{\partial x}(\lambda+ \\
& 2 \mu+\mathrm{P})+\mathrm{u}_{3, \mathrm{z}} \frac{\partial}{\partial x}(\lambda+\mathrm{P})+\left(\mathrm{u}_{3, \mathrm{x}}+\mathrm{u}_{1, \mathrm{z}}\right) \frac{\partial}{\partial z}(\mu)-\rho \mathrm{g} \mathrm{u}_{3, \mathrm{x}}-\mathrm{F} \\
& \frac{\partial}{\partial t}\left(\eta_{, \mathrm{z}}\right)-\frac{\partial \eta}{\partial t} \frac{\partial}{\partial z}(\mathrm{~F})-\frac{P}{2}\left(\mathrm{u}_{1, \mathrm{zz}}-\mathrm{u}_{3, \mathrm{xz}}\right)+\mu_{e} H_{0}^{2} \\
& \left(2 \mathrm{u}_{1, \mathrm{xx}}+\mathrm{u}_{3, \mathrm{xz}}\right)-\Xi \frac{\partial T}{\partial x}=\rho \mathrm{u}_{1, \mathrm{tt}}, \\
& \left(\mathrm{F} \xi_{, \mathrm{t}}\right)_{, \mathrm{z}}-\left(\mathrm{F} \zeta_{, \mathrm{t}}\right), \mathrm{x}=0 \\
& (\lambda+2 \mu) u_{3, z z}+\mu u_{3, x x}+(\lambda+\mu) u_{1, x z}+\left(u_{3, x}+u_{1, z}\right) \frac{\partial}{\partial x}(\mu) \\
& +\mathrm{u}_{1, \mathrm{x}} \frac{\partial}{\partial z}(\lambda)+\mathrm{u}_{3, \mathrm{z}} \frac{\partial}{\partial z}(\lambda+2 \mu)+\mu_{e} H_{0}^{2}\left(\mathrm{u}_{1, \mathrm{xz}}-\mathrm{u}_{3, \mathrm{xx}}\right) \\
& -\frac{P}{2}\left(\mathrm{u}_{1, \mathrm{xz}}-\mathrm{u}_{3, \mathrm{xx}}\right)+\left(\mathrm{u}_{1, \mathrm{x}}+\mathrm{u}_{3, \mathrm{z}}\right) \mu_{e} H_{0}^{2}+\rho \mathrm{g} \mathrm{u}_{1, \mathrm{x}}-\Xi \frac{\partial T}{\partial z}+ \\
& \mathrm{F} \frac{\partial}{\partial t}\left(\eta_{, \mathrm{x}}\right)=\rho \mathrm{u}_{3, \mathrm{tt}}, \\
& -F \frac{\partial \xi}{\partial t}+M \nabla^{2} \xi+\xi, \mathrm{z} \frac{\partial}{\partial z}(\mathrm{M})=0, \\
& -F \frac{\partial \eta}{\partial t}+M \nabla^{2}\left(\eta+w_{2}\right)+\frac{\partial}{\partial z}\left(w_{2}+\eta\right) \frac{\partial M}{\partial z}=0,(11 \mathrm{a}) \\
& -F \frac{\partial \mathrm{z}}{\partial t}+\mathrm{M} \nabla^{2}+\zeta_{, z} \frac{\partial(M)}{\partial z}=0,
\end{aligned}
$$

where $\lambda, \mu$ are Lame's constants and

$$
\Delta=\frac{\partial u_{1}}{\partial x}+\frac{\partial u_{3}}{\partial z}=u_{1, x}+u_{3, z}
$$

Now we assume the non-homogeneity of the granular half-space and co-efficient of friction are given by

$$
\begin{gathered}
\lambda=\lambda_{0} \mathrm{e}^{\mathrm{mz}}, \mu=\mu_{0} \mathrm{e}^{\mathrm{mz}}, \rho=\rho_{0} \mathrm{e}^{\mathrm{mz}}, \mathrm{F}=\mathrm{F}_{0} \mathrm{e}^{\mathrm{mz}}, \\
\mathrm{M}=\mathrm{M}_{0} \mathrm{e}^{\mathrm{mz}}, \mathrm{P}=\mathrm{P}_{0} \mathrm{e}^{\mathrm{mz}}, \mu_{\mathrm{e}}=\left(\mu_{\mathrm{e}}\right)_{0} \mathrm{e}^{\mathrm{mz}} \Xi=\Xi_{0} \mathrm{e}^{\mathrm{mz}}
\end{gathered}
$$

Where $\mathrm{m}, \lambda_{0}, \mu_{0}, \rho_{0}, \mathrm{~F}_{0}, \mathrm{M}_{0}, \mathrm{P}_{0},\left(\mu_{\mathrm{e}}\right)_{0}, \Xi_{0}$ are dimensionless constants.

Inserting Inhomogeneities in Eq. (11), we get

$$
\begin{array}{r}
\left(\lambda_{0}+2 \mu_{0}+\mathrm{P}_{0}\right) \mathrm{u}_{1, \mathrm{xx}}+\mu_{0} \mathrm{u}_{1, \mathrm{zz}}+\left(\lambda_{0}+\mu_{0}+\mathrm{P}_{0}\right) \mathrm{u}_{3, \mathrm{xz}}+\mathrm{u}_{1, \mathrm{x}} \frac{\partial}{\partial x} \\
\left(\lambda_{0}+2 \mu_{0}+\mathrm{P}_{0}\right)+\mathrm{u}_{3, \mathrm{z}} \frac{\partial}{\partial x}\left(\lambda_{0}+\mathrm{P}_{0}\right)+\left(\mathrm{u}_{3, \mathrm{x}}+\mathrm{u}_{1, \mathrm{z}}\right) \frac{\partial}{\partial z}\left(\mu_{0}\right)- \\
\rho_{0} \mathrm{gu}_{3, \mathrm{x}}-\mathrm{F}_{0} \frac{\partial}{\partial t}\left(\eta_{, z}\right)-\frac{\partial \eta}{\partial t} \frac{\partial}{\partial z}\left(\mathrm{~F}_{0}\right)+\left(\mu_{e}\right)_{0} H_{0}^{2}
\end{array}
$$




$$
\begin{gathered}
\left(2 \mathrm{u}_{1, \mathrm{xx}}+\mathrm{u}_{3, \mathrm{xz}}\right)-\frac{P_{0}}{2}\left(\mathrm{u}_{1, \mathrm{zz}}-\mathrm{u}_{3, \mathrm{xz}}\right)-\Xi_{0} \frac{\partial T}{\partial x}=\rho_{0} \mathrm{u}_{1, \mathrm{tt}}, \\
\therefore\left(\mathrm{F}_{0} \xi_{, \mathrm{t}}\right)_{, \mathrm{z}}-\left(\mathrm{F}_{0} \zeta_{, \mathrm{t}}\right)_{\mathrm{x}}=0, \\
\left(\lambda_{0}+2 \mu_{0}\right) \mathrm{u}_{3, \mathrm{zz}}+\mu_{0} \mathrm{u}_{3, \mathrm{xx}}+\left(\lambda_{0}+\mu_{0}\right) \mathrm{u}_{1, \mathrm{xz}}+\left(\mathrm{u}_{3, \mathrm{x}}+\mathrm{u}_{1, \mathrm{z}}\right) \frac{\partial}{\partial x} \\
\left(\mu_{0}\right)+\mathrm{u}_{1, \mathrm{x}} \frac{\partial}{\partial z}\left(\lambda_{0}\right)+\mathrm{u}_{3, \mathrm{z}} \frac{\partial}{\partial z}\left(\lambda_{0}+2 \mu_{0}\right)+\left(\mu_{e}\right)_{0} H_{0}^{2} \\
\left(\mathrm{u}_{1, \mathrm{xz}}-\mathrm{u}_{3, \mathrm{xx}}\right)-\frac{P_{0}}{2}\left(\mathrm{u}_{1, \mathrm{xz}}-\mathrm{u}_{3, \mathrm{xx}}\right)+\left(\mathrm{u}_{1, \mathrm{x}}+\mathrm{u}_{3, \mathrm{z}}\right)+\left(\mu_{e}\right)_{0} H_{0}^{2} \\
+\rho_{0} \mathrm{~g} \mathrm{u}_{1, \mathrm{x}}-\Xi_{0} \frac{\partial T}{\partial z}+\mathrm{F}_{0} \frac{\partial}{\partial t}\left(\eta_{, \mathrm{x}}\right)=\rho_{0} \mathrm{u}_{3, \mathrm{t}}, \\
-F_{0} \frac{\partial \xi}{\partial t}+\mathrm{M}_{0} \nabla^{2} \xi+\xi_{, \mathrm{z}} \frac{\partial}{\partial z}\left(\mathrm{M}_{0}\right)=0, \\
-F_{0} \frac{\partial \eta}{\partial t}+\mathrm{M}_{0} \nabla^{2}\left(\eta+\mathrm{w}_{2}\right)+\frac{\partial}{\partial z}\left(w_{2}+\eta\right) \frac{\partial M_{0}}{\partial z}=0,(11 \mathrm{~b}) \\
-F_{0} \frac{\partial \mathrm{z}}{\partial t}+\mathrm{M}_{0} \nabla^{2} \zeta \\
+\zeta, \frac{\partial\left(M_{0}\right)}{\partial z}=0,
\end{gathered}
$$

To determine T, Fourier's law of heat conduction

$$
\mathrm{p} \nabla^{2} \mathrm{~T}=C_{v} \frac{\partial T}{\partial t}+T_{0} G_{L} \frac{\partial}{\partial t}\left(\nabla^{2} \phi\right),
$$

where $\mathrm{K}$ be the thermal conductivity and obeys the law as given by $\mathrm{K}=\mathrm{K}_{0} \mathrm{e}^{\mathrm{mz}}$,

$\mathrm{p}=\frac{K_{0}}{\rho_{0}}$ and $\mathrm{C}$ be the specific heat of the body at constant volume.

Further to investigate the surface wave propagation, we introduce displacement potentials in terms of displacement components are given by

$$
\mathrm{u}_{1}=\phi_{, \mathrm{x}}-\psi_{, \mathrm{z}}, \mathrm{u}_{3}=\phi_{, \mathrm{z}}+\psi_{, \mathrm{x}}
$$

Introducing Eq. (12), (13) into Eq. (11b), we get

$$
\begin{gathered}
\alpha^{2} \nabla^{2} \phi-\phi_{, \mathrm{tt}}+\mathrm{g} \psi_{, \mathrm{x}}+\mathrm{m} \beta^{\prime^{2}}\left(2 \phi_{, \mathrm{z}}+\psi_{, \mathrm{x}}\right)-\delta^{2} \mathrm{~T}=0, \\
\frac{\partial}{\partial t}\left(\xi_{, \mathrm{z}}-\zeta_{, \mathrm{x}}\right)+\mathrm{m} \xi_{, \mathrm{t}}=0, \\
\beta^{2} \nabla^{2} \psi-\psi_{, \mathrm{tt}}+\mathrm{g} \phi_{, \mathrm{x}}+\mathrm{s}_{\eta, \mathrm{t}}+\mathrm{m}\left(\gamma^{2} \phi_{, \mathrm{x}}+2 \beta^{2} \psi_{, \mathrm{z}}\right)=0, \\
-\mathrm{F}_{0} \xi_{, \mathrm{t}}+\mathrm{M}_{0} \nabla^{2} \xi+\mathrm{M}_{0} \mathrm{~m} \xi_{, \mathrm{z}}=0, \\
-\mathrm{s}_{\eta_{, \mathrm{t}}}+\nabla^{2} \eta-\nabla^{4} \psi-\mathrm{m}\left[\eta_{, \mathrm{z}}-\nabla^{2}\left(\psi_{, \mathrm{z}}\right)\right]=0,
\end{gathered}
$$

$$
-\mathrm{s}^{\prime} \xi_{, \mathrm{t}}+\nabla^{2} \xi+\mathrm{m} \zeta_{, \mathrm{z}}=0,
$$

where

$$
\begin{gathered}
\frac{\lambda_{0}+2 \mu_{0}+P_{0}+\left(\mu_{e}\right)_{0} H_{0}^{2}}{\rho_{0}}=\alpha^{2}, \\
\beta^{2}=\frac{2 \mu_{0}+P_{0}}{2 \rho_{0}}, \gamma^{2}=\frac{\lambda_{0}+\left(\mu_{e}\right)_{0} H_{0}^{2}}{\rho_{0}}, \\
\mathrm{~s}=\frac{F_{0}}{\rho_{0}}, \delta^{2}=\frac{K_{0}}{\rho_{0}}, \mathrm{~s}^{\prime}=\frac{F_{0}}{M_{0}}, \beta^{2}=\beta^{2}-\frac{P_{0}}{2 \rho_{0}}
\end{gathered}
$$

Eliminating $\eta$ from Eq. (16) and Eq. (18); we get

$$
\begin{aligned}
& \left(\nabla^{2}-s^{\prime} \frac{\partial}{\partial t}+m \frac{\partial}{\partial z}\right)\left[\beta^{2} \nabla^{2} \psi-\psi_{, \mathrm{tt}}+\mathrm{g} \phi_{, \mathrm{x}}+\mathrm{m}\left(\gamma^{2}\right.\right. \\
& \left.\left.\phi, \mathrm{x}+2 \beta^{2} \psi, \mathrm{z}\right)\right]+\mathrm{s} \nabla^{4}\left(\psi_{, \mathrm{t}}\right)+\mathrm{ms} \nabla^{2}(\psi, \mathrm{zt})=0 .(21)
\end{aligned}
$$

To solve Eq. (14) to Eq. (19), we assume that

$$
\begin{aligned}
& \phi(x, z, t)=\phi_{1}(z) e^{i(l x-b t)}, \\
& \psi(x, z, t)=\psi_{1}(z) e^{i(l x-b t)}, \\
& \xi(x, z, t)=\xi_{1}(z) e^{i(l x-b t)}, \\
& \eta(x, z, t)=\eta_{1}(z) e^{i(l x-b t)}, \\
& \zeta(x, z, t)=\zeta_{1}(z) e^{i(l x-b t)} .
\end{aligned}
$$

putting Eq. (22) in Eq. (14) and Eq. (21), we get

$$
\begin{gathered}
\left(\alpha^{2} \mathrm{D}^{2}-\mathrm{A}\right) \phi_{1}-\mathrm{B} \psi_{1}=0, \\
\left(\mathrm{~A}^{\prime} \mathrm{D}^{4}+\mathrm{B}^{\prime} \mathrm{D}^{3}+\mathrm{C}^{\prime} \mathrm{D}^{2}+\mathrm{d}^{\prime} \mathrm{D}\right. \\
+\mathrm{E}) \psi_{1}+\left(\mathrm{E}^{\prime} \mathrm{D}^{2}+\mathrm{F}^{\prime}\right) \phi_{1}=0,
\end{gathered}
$$

Where

$$
\begin{gathered}
\mathrm{D}=\frac{d}{d z}, \mathrm{~A}=\alpha^{2} l^{2}-\mathrm{b}^{2}-2 \mathrm{~m} \beta^{2}, \\
\mathrm{~B}=\mathrm{i} l \mathrm{~g}-\mathrm{i} l \mathrm{~m} \beta^{2}, \\
\mathrm{~A}^{\prime}=\beta^{2}-\mathrm{ibs}, \mathrm{B}^{\prime}=3 \mathrm{~m} \beta^{2}-\mathrm{imsb}, \\
\mathrm{C}^{\prime}=\mathrm{b}\left(\mathrm{b}+\mathrm{i} \beta^{2} \mathrm{~s}^{\prime}\right)-2 l^{2}\left(\mathrm{~B}^{2}-\mathrm{ibs}\right)+2 \mathrm{~m}^{2} \beta^{2}, \\
\mathrm{~d}^{\prime}=\left(-2 \beta^{2} l^{2} \mathrm{~m}+2 \beta^{2} \mathrm{is} \mathrm{s}^{\prime} \mathrm{m}-\mathrm{m} l^{2} \beta^{2}+\mathrm{mb} b^{2}+\mathrm{imsb} l^{2}\right), \\
\mathrm{E}=l^{4}\left(\beta^{2}-\mathrm{ibs}\right)-\mathrm{b} l^{2}\left(\mathrm{~b}+\mathrm{i} \beta^{2} \mathrm{~s}^{\prime}\right)+\mathrm{ib} \mathrm{s}^{\prime}, \mathrm{E}^{\prime}=\left(\mathrm{ilg}+\mathrm{im} l \gamma^{2}\right), \\
\mathrm{F}^{\prime}=\left(-\mathrm{ig} l^{3}-\mathrm{g} l \mathrm{bs} \mathrm{s}^{\prime}-\mathrm{im} l^{3} \gamma^{2}-\mathrm{ms} \gamma^{2} \mathrm{~b}^{2}+\mathrm{i} l \mathrm{gm}+\mathrm{i} l \mathrm{~m}^{2} \gamma^{2}\right) .(25)
\end{gathered}
$$


Therefore the solutions of Eq. (23) and Eq. (24) is of the form

$$
\begin{array}{r}
\phi_{1}=A_{j} e^{\lambda_{j} z}+B_{j} e^{-\lambda_{j} z} \\
\psi_{1}=E_{j} e^{\lambda_{j} z}+F_{j} e^{-\lambda_{j} z}, \mathrm{j}=3,4,5
\end{array}
$$

where

$\mathrm{j}(\mathrm{j}=3,4,5)$ are the real roots of the following equation

$$
\mathrm{D}^{6}+\mathrm{P}_{1} \mathrm{D}^{5}+\mathrm{P}_{2} \mathrm{D}^{4}+\mathrm{P}_{3} \mathrm{D}^{3}+\mathrm{P}_{4} \mathrm{D}^{2}+\mathrm{P}_{5} \mathrm{D}+\mathrm{P}_{6}=0
$$

Where,

$$
\begin{gathered}
\mathrm{P}_{1}=\frac{3 m \beta^{2}-i m s b}{\beta^{2}-i b s}, \\
P_{2}=\frac{\left[\begin{array}{l}
\alpha^{2} b\left(b+i \beta s^{\prime}\right)-2 l^{2} \alpha^{2}\left(\beta^{2}-i b s\right) \\
+2 \alpha^{2} m^{2} \beta^{2} \\
-\left(\beta^{2}-i b s\right)\left(\alpha^{2} l^{2}-b^{2}-2 m \beta^{2}\right)
\end{array}\right]}{\alpha^{2}\left(\beta^{2}-i b s\right)} \\
P_{3}^{2}\left(\begin{array}{l}
\left.-2 \beta^{2} l^{2}+2 \beta^{2} i s^{\prime}-m l^{2} \beta^{2}+m b^{2}+i m s b l^{2}\right) \\
\left.\alpha^{2} l^{2}-b^{2}-2 m \beta^{2}\right)\left(3 m \beta^{2}-i m s b\right) \\
\mathrm{P}_{4}=\frac{\mathrm{P}_{6}=\frac{\left.\beta^{2}-i b s\right)}{\alpha^{2}\left(\beta^{2}-i b s\right)}}{\alpha^{2}\left(\beta^{2}-i b s\right)}, \mathrm{P}_{5}=\frac{-A d^{\prime}}{\alpha^{2}\left(\beta^{2}-i b s\right)}
\end{array},\right.
\end{gathered}
$$

where A', B', C', D', E', E, F, A, B are given by Eq. (25).

Further the constants $A_{i}, B_{j}(j=3,4,5)$ are related with constants $E_{j}, F_{j}$ respectively by means of Eq. (23). Equating the coefficients of $\mathrm{e}^{\lambda \mathrm{jz}}, \mathrm{e}^{-\lambda \mathrm{z}}(\mathrm{j}=3,4,5)$ to zero and using Eq. (23) and (24); we get

Where,

$$
\begin{gathered}
\mathrm{A}_{\mathrm{j}}=\eta_{\mathrm{j}} \mathrm{E}_{\mathrm{j}} \text { and } \mathrm{B}_{\mathrm{j}}=\eta_{\mathrm{j}} \mathrm{F}_{\mathrm{j}}(\mathrm{j}=3,4,5), \\
\eta_{\mathrm{j}}=\frac{i \lg -i l m \beta^{2}}{\alpha^{2} \lambda j^{2}-\alpha^{2} l^{2}+b^{2}+2 m \beta^{2}}(\mathrm{j}=3,4,5)(31)
\end{gathered}
$$

Now solving Eq. (16) and Eq. (23) for $\eta_{1}$ and $\psi_{1}$, we get

$$
\left(a_{1} D^{4}+a_{2} D^{3}+a_{3} D^{2}+a_{4} D+a_{5}\right) \psi_{1}
$$

$$
- \text { isb }\left(\alpha^{2} D^{2}+a_{6}\right) \eta_{1}=0
$$

Now eliminating $\psi_{1}$ from Eq. (18) and Eq. (32), we get

$$
\begin{aligned}
& {\left[q_{1} D^{6}+q_{2} D^{5}+q_{3} D^{4}+q_{4} D^{3}\right.} \\
& \left.\quad+q_{5} D^{2}+q_{6} D+q_{7}\right] \eta=0
\end{aligned}
$$

Where

$$
\begin{aligned}
& \mathrm{q}_{1}=\mathrm{a}_{1}-\mathrm{isb} \alpha^{2} \\
& \mathrm{q}_{2}=\mathrm{ma} \mathrm{a}_{1}+\mathrm{a}_{2}-\mathrm{isb} \alpha^{2} \mathrm{~m} \text {, } \\
& \mathrm{q}_{3}=\mathrm{a}_{1}\left(\mathrm{is} \mathrm{b}-l^{2}\right)+\mathrm{a}_{2} \mathrm{~m}+\mathrm{a}_{3}-\mathrm{isba}_{6}+2 \mathrm{isb} l^{2} \alpha^{2}, \\
& \mathrm{q}_{4}=\mathrm{a}_{2}\left(\mathrm{is} \mathrm{s}^{\prime} \mathrm{b}-l^{2}\right)+\mathrm{a}_{3} \mathrm{~m}+\mathrm{a}_{4}-\text { isbma }_{6}+\mathrm{isbm} l^{2} \alpha^{2}, \\
& \mathrm{q}_{5}=\mathrm{a}_{3}\left(\text { is' } \mathrm{b}-l^{2}\right)+\mathrm{a}_{4} \mathrm{~m}+\mathrm{a}_{5}+2 \text { isb } l^{2} \mathrm{a}_{6}+\alpha^{2} l^{4}(-\mathrm{isb}), \\
& \mathrm{q}_{6}=\left(\text { is' } \mathrm{b}-l^{2}\right) \mathrm{a}_{4}+\mathrm{ma}_{5}+\text { isb } \mathrm{m} l^{2} \mathrm{a}_{6}, \\
& \mathrm{q}_{7}=\mathrm{a}_{5}\left(\text { is' } \mathrm{b}-l^{2}\right)-\mathrm{isb} l^{4} \mathrm{a}_{6}, \\
& a_{1}=\alpha^{2} \beta^{2} \\
& \mathrm{a}_{2}=2 \mathrm{~m} \alpha^{2} \beta^{2} \text {, } \\
& \mathrm{a}_{3}=\beta^{2}\left(-\alpha^{2} l^{2}-\mathrm{b}^{2}+2 \mathrm{~m} \beta^{2}\right)+\left(\mathrm{b}^{2}-l^{2} \beta^{2}\right) \alpha^{2}, \\
& \mathrm{a}_{4}=2 \mathrm{~m} \beta^{2}\left(-\alpha^{2} l^{2}+\mathrm{b}^{2}+2 \mathrm{~m} \beta^{2}\right), \\
& \mathrm{a}_{5}=\left(\mathrm{b}^{2}-l^{2} \beta^{2}\right)\left(-\alpha^{2} l^{2}+\mathrm{b}^{2}+2 \mathrm{~m} \beta^{2}\right)+\mathrm{i} l\left(\mathrm{~g}+\mathrm{m} \gamma^{2}\right)(\mathrm{i} l \mathrm{~g}- \\
& \left.\mathrm{i} l \mathrm{~m} \beta^{2}\right) \text {, } \\
& \mathrm{a}_{6}=\mathrm{b}^{2}+2 \mathrm{~m} \beta^{2}-\alpha^{2} l^{2} \text {. }
\end{aligned}
$$

The solution of Eq. (33) is of the form

$$
\eta_{\mathrm{j}}=\left(E_{j} e^{\lambda_{j} z}+F_{j} e^{-\lambda_{j} z}\right) \delta_{\mathrm{j}}
$$

where $\lambda_{j}(j=3,4,5)$ are the real roots of Eq. (33) and

$\delta_{\mathrm{j}}=\frac{-i}{b s}\left[\beta^{2}\left(\lambda_{\mathrm{j}}^{2}-l^{2}\right)+\mathrm{b}^{2}+\left(\mathrm{i} l \mathrm{~g}+\mathrm{mi} l \gamma^{2}\right) \mathrm{n}_{\mathrm{j}}+2 \mathrm{~m} \beta^{2} \lambda_{\mathrm{j}}\right]$

where $\mathrm{j}=3,4,5$ and $\mathrm{n}_{\mathrm{j}}$ is given by Eq. (31).

Further substituting Eq. (22) into Eq. (15), Eq. (17) and Eq. (19), we get

$$
\begin{aligned}
& (\mathrm{D}+\mathrm{m}) \xi_{1}-\mathrm{i} l \zeta_{1}=0 \\
& \left(\mathrm{D}^{2}+\mathrm{mD}+\mathrm{h}^{2}\right) \xi_{1}=0 \\
& \left(\mathrm{D}^{2}+\mathrm{mD}+\mathrm{h}^{2}\right) \zeta_{1}=0
\end{aligned}
$$


Where

$$
\mathrm{h}^{2}=\mathrm{is} \mathrm{b}-l^{2},
$$

The solutions of Eq. (38) and Eq. (39) are given by

$$
\begin{aligned}
& \xi_{1}=A_{1} e^{\alpha z}+A z e^{-\beta z}, \\
& \zeta_{1}=B_{1} e^{\alpha z}+B_{2} z e^{-\beta z},
\end{aligned}
$$

where

$$
\begin{gathered}
\alpha=\frac{-m+\sqrt{m^{2}-4 h^{2}}}{2}, \\
\beta=\frac{m+\sqrt{m^{2}-4 h^{2}}}{2}, \mathrm{~m}^{2}-4 \mathrm{~h}^{2}>0 .
\end{gathered}
$$

Substituting Eq. (40), Eq. (41) into Eq. (37), we get

$$
\begin{aligned}
& \left(A_{1} \alpha+A_{1} m\right) e^{\alpha z}+\left(-A_{2} \beta+\right. \\
& \left.m A_{2}\right) e^{-\beta z}=i l\left(B_{1} e^{\alpha z}+B_{2} e^{-\beta z}\right) .
\end{aligned}
$$

Equating the co-efficients of $\mathrm{e}^{\alpha \mathrm{z}}$ and $\mathrm{e}^{-\beta \mathrm{z}}$ to zero in Eq. (42), we get

$$
\mathrm{A}_{1}=\frac{i l B_{1}}{\alpha+m}, \mathrm{~A}_{2}=\frac{i l B_{2}}{m-\beta}
$$

Let $\lambda_{0}, \mu_{0}, \rho_{0}, \mathrm{~F}_{0}, \mathrm{M}_{0}$ are the characteristics of layer and $\bar{\lambda}_{0}, \bar{\mu}_{0}, \bar{\rho}_{0}, \bar{F}_{0}, \bar{M}_{0}$ are the characteristics of half-space, also for the lower half-space and description of surface wave propagation $\xi_{1}, \phi_{1}, \eta_{1}, \psi_{1}, \zeta_{1}$ goes to zero as $\mathrm{z} \rightarrow \infty$, also the non-homogeneity constant $\mathrm{m}$ is replaced by constant $\bar{m}$ for lower granular half-space also it is assumed that the real parts of $(j=3,4,5)$ are positive.

Thus for lower half-space

$$
\begin{gathered}
\bar{\phi}_{1}=\bar{\eta}_{j} \bar{F}_{j} e^{-\bar{\lambda}_{j} z}, \\
\bar{\psi}_{1}=\bar{F}_{j} e^{-\bar{\lambda}_{j z}}, \\
\bar{\eta}_{1}=\bar{\delta}_{j} \bar{F}_{j} e^{-\bar{\lambda}_{j} z}, \\
\bar{\xi}_{1}=\frac{i l}{\bar{m}-\bar{\beta}} \bar{B}_{2} e^{-\bar{\beta} z}, \\
\overline{\mathrm{Z}}_{1}=\bar{B}_{2} e^{-\bar{\beta} z}(\mathrm{j}=3,4,5) .
\end{gathered}
$$

\section{Boundary Conditions and Dispersion Equation}

Case-I The boundary conditions on interface $z=H$ are

(i) $\mathrm{u}_{1}=\bar{u}_{1}$,

(ii) $\mathrm{u}_{3}=\bar{u}_{3}$,

(iii) $\xi=\bar{\xi}$,

(iv) $\eta=\bar{\eta}$

(v) $\zeta=\bar{z}$,

(vi) $\mathrm{M}_{33}=\bar{M}_{33}$,

(vii) $\mathrm{M}_{31}=\bar{M}_{31}$,

(viii) $\mathrm{M}_{32}=\bar{M}_{32}$,

(ix) $\sigma_{33}=\bar{\sigma}_{33}$,

(x) $\sigma_{31}=\bar{\sigma}_{31}$,

(xi) $\sigma_{32}=\bar{\sigma}_{32}$.

(xii) $\mathrm{T}=\bar{T}$

(xiii) $\frac{\partial T}{\partial z}+\theta T=\frac{\partial \bar{T}}{\partial z}+\theta \bar{T}$

Case-II The boundary conditions on free surface $z=0$ are

$$
\begin{aligned}
& \left(\text { xii) } M_{33}=0\right. \\
& \text { (xiii) } M_{31}=0 \\
& \text { (xiv) } M_{32}=0, \\
& \text { (xv) } \sigma_{33}=0 \\
& \text { (xvi) } \sigma_{31}=0 \\
& \text { (xvii) } \sigma_{32}=0
\end{aligned}
$$

where

$$
\begin{gathered}
M_{31}=M_{0} e^{m z} \xi, z, \\
M_{32}=M_{0} e^{m z}\left(\eta-\nabla^{2} \psi\right), z \\
M_{33}=M_{0} e^{m z} \zeta, z,
\end{gathered}
$$




$$
\begin{gathered}
\sigma_{31}=\mu_{0} \mathrm{e}^{\mathrm{mz}}\left(2 \phi_{, \mathrm{xz}}-\psi_{, \mathrm{zz}}+\psi_{, \mathrm{xx}}\right)-\mathrm{F}_{0} \mathrm{e}^{\mathrm{mz}} \eta, \mathrm{t}, \\
\sigma_{32}=-\mathrm{F}_{0} \mathrm{e}^{\mathrm{mz}} \xi_{, \mathrm{t}}, \\
\sigma_{33}=\lambda_{0} \mathrm{e}^{\mathrm{mz}} \nabla^{2} \phi+2 \mu\left(\phi_{, \mathrm{zz}}+\psi_{, \mathrm{xz}}\right) .
\end{gathered}
$$

From the boundary conditions (iii), (v), (vi) and (vii), we get

$$
\begin{gathered}
\frac{B_{1}}{\alpha+m} e^{\alpha H}+\frac{B_{2}}{m-\beta} e^{-\beta H}=\frac{\bar{B}_{2}}{\bar{m}-\bar{\beta}} e^{-\bar{\beta} H} \\
\mathrm{~B}_{1} \mathrm{e}^{\alpha \mathrm{H}}+\mathrm{B}_{2} \mathrm{e}^{-\beta \mathrm{H}}=\bar{B}_{2} e^{-\bar{\beta} H} \\
\mathrm{M}_{0} \mathrm{e}^{\mathrm{mH}}\left[\mathrm{B}_{1} \alpha \mathrm{e}^{\alpha \mathrm{H}}-\mathrm{B}_{2} \beta \mathrm{e}^{-\beta \mathrm{H}}\right]= \\
-\bar{M}_{0} e^{\bar{m} H} \bar{\beta} \bar{B}_{2} e^{-\bar{\beta} H} \\
M_{0} e^{m H}\left[\frac{B_{1} \alpha e^{\alpha H}}{\alpha+m}-\frac{B_{2} \beta e^{-\beta H}}{m-\beta}\right]= \\
-\bar{M}_{0} e^{\bar{m} H} \frac{\bar{\beta} \bar{B}_{2}}{\bar{m}-\bar{\beta}} e^{-\bar{\beta} H}
\end{gathered}
$$

From Eq. (52) to Eq. (55), we have

$$
\begin{gathered}
\mathrm{B}_{1}=\mathrm{B}_{2}=\bar{B}_{2}=0 \\
\text { i.e. } \xi=\zeta=\overline{\mathrm{z}}=\bar{\xi}=0 .
\end{gathered}
$$

The other boundary conditions gives the following relations, conditions (xii) and (xiii) are identities due to Eq. (57).

(xiv) gives, i.e. $\mathrm{K}_{1} \mathrm{E}_{3}+\mathrm{K}_{2} \mathrm{E}_{4}+\mathrm{K}_{3} \mathrm{E}_{5}-\mathrm{K}_{1} \mathrm{~F}_{3}-\mathrm{K}_{2}$

$$
\mathrm{F}_{4}-\mathrm{K}_{3} \mathrm{~F}_{5}=0 \text {, }
$$

(xv) gives, $\mathrm{K}_{4} \mathrm{E}_{3}+\mathrm{K}_{5} \mathrm{E}_{4}+\mathrm{K}_{6} \mathrm{E}_{5}+\mathrm{K}_{7} \mathrm{~F}_{3}+\mathrm{K}_{8} \mathrm{~F}_{4}$

$$
+\mathrm{K}_{9} \mathrm{~F}_{3}=0
$$

(xvi) gives, $\mathrm{K}_{10} \mathrm{E}_{3}+\mathrm{K}_{11} \mathrm{E}_{4}+\mathrm{K}_{12} \mathrm{E}_{5}+\mathrm{K}_{13} \mathrm{~F}_{3}+$

$$
\mathrm{K}_{14} \mathrm{~F}_{4}+\mathrm{K}_{15} \mathrm{~F}_{5}=0 \text {, }
$$

while condition (xviii) and (xi) is an identity,

(i) gives, $\mathrm{K}_{16}$

$$
\begin{array}{r}
e^{\lambda_{3} H} E_{3}+K_{17} e^{\lambda_{4} H} E_{4}+K_{18} e^{\lambda_{5} H} E_{5}+K_{19} e^{-\lambda_{3} H} F_{3} \\
+K_{20} e^{-\lambda_{4} H} F_{4}+K_{21} e^{-\lambda_{5} H} F_{5}= \\
\bar{K}_{19} e^{-\bar{\lambda}_{3} H} \bar{F}_{3}+\bar{K}_{20} e^{-\bar{\lambda}_{4} H} \bar{F}_{4}+\bar{K}_{21} e^{-\bar{\lambda}_{5} H} \bar{F}_{5},
\end{array}
$$

(ii) gives (il $\left.+\mathrm{n}_{3} \lambda_{3}\right) e^{\lambda_{3} H} \mathrm{E}_{3}+\left(\mathrm{i} l+\mathrm{n}_{4} \lambda_{4}\right) e^{\lambda_{4} H} \mathrm{E}_{4}$ $+\left(\mathrm{i} l+\mathrm{n}_{5} \lambda_{5}\right) e^{\lambda_{5} H} \mathrm{E}_{5}$

$+\left(\mathrm{i} l-\mathrm{n}_{3} \lambda_{3}\right) e^{-\lambda_{3} H} \quad \mathrm{~F}_{3}+\left(\mathrm{i} l-\mathrm{n}_{4} \lambda_{4}\right) e^{-\lambda_{4} H} \mathrm{~F}_{4}+(\mathrm{i} l$ $\left.-\mathrm{n}_{5} \lambda_{5}\right) e^{-\lambda_{5} H} \mathrm{~F}_{5}$

$$
\begin{aligned}
& \left(i l-\bar{n}_{3} \bar{\lambda}_{3}\right) e^{-\bar{\lambda}_{3} H} \bar{F}_{3} \\
& +\left(i l-\bar{n}_{4} \bar{\lambda}_{4}\right) e^{-\bar{\lambda}_{4} H} \bar{F}_{4}+\left(i l-\bar{n}_{5} \bar{\lambda}_{5}\right) e^{-\bar{\lambda}_{5} H} \bar{F}_{5},
\end{aligned}
$$

(iv) gives, $\delta_{3} e^{\lambda_{3} H} \mathrm{E}_{3}+\delta_{4} e^{\lambda_{4} H} \mathrm{E}_{4}+\delta_{5} e^{\lambda_{5} H} \mathrm{E}_{5}+$

$$
\begin{gathered}
\delta_{3} e^{-\lambda_{3} H} \mathrm{~F}_{3} \\
+\delta_{4} e^{-\lambda_{4} H} F_{4}+\delta_{5} e^{-\lambda_{5} H} F_{5}= \\
\bar{\delta}_{3} e^{-\bar{\lambda}_{3} H} \bar{F}_{3}+\bar{\delta}_{4} e^{-\bar{\lambda}_{4} H} \bar{F}_{4}+\bar{\delta}_{5} e^{-\bar{\lambda}_{5} H} \bar{F}_{5},
\end{gathered}
$$

(viii) gives, $\mathrm{M}_{0} \mathrm{e}^{\mathrm{mH}}$

$$
\begin{aligned}
& {\left[K_{1} e^{\lambda_{3} H} E_{3}+K_{2} e^{\lambda_{4} H} E_{4}+K_{3} e^{\lambda_{5} H} E_{5}-K_{1} e^{-\lambda_{3} H}\right.} \\
& \left.F_{3}-K_{2} e^{-\lambda_{4} H} F_{4}-K_{3} e^{-\lambda_{5} H} F_{5}\right] \\
& =-\bar{M}_{0} e^{\bar{m} H}\left[\begin{array}{l}
\bar{K}_{1} e^{-\bar{\lambda}_{3} H} \bar{F}_{3}+ \\
\bar{K}_{2} e^{-\bar{\lambda}_{4} H} \bar{F}_{4}+\bar{K}_{3} e^{-\bar{\lambda}_{5} H} \bar{F}_{5}
\end{array}\right],
\end{aligned}
$$

(ix) gives, $\mathrm{e}^{\mathrm{mH}}$

$$
\begin{array}{r}
{\left[K_{4} e^{\lambda_{3} H} E_{3}+K_{5} e^{\lambda_{4} H} E_{4}+K_{6} e^{\lambda_{5} H} E_{5}+K_{7} e^{-\lambda_{3} H} F_{3}\right.} \\
\left.+K_{8} e^{-\lambda_{4} H} F_{4}+K_{9} e^{-\lambda_{5} H} F_{5}\right]= \\
e^{\bar{m} H}\left[\bar{K}_{7} e^{-\bar{\lambda}_{3} H} F_{3}+\bar{K}_{8} e^{-\bar{\lambda}_{4} H} \bar{F}_{4}+\bar{K}_{9} e^{-\bar{\lambda}_{5} H} \bar{F}_{5}\right],
\end{array}
$$

(x) gives, $\mathrm{e}^{\mathrm{mH}}$

$$
\begin{aligned}
& {\left[\begin{array}{c}
K_{10} e^{\lambda_{3} H} E_{3}+K_{11} e^{\lambda_{4} H} E_{4}+ \\
K_{12} e^{\lambda_{5} H} E_{5}+K_{13} e^{-\lambda_{3} H} F_{3}
\end{array}\right.} \\
+ & \left.K_{14} e^{-\lambda_{4} H} F_{4}+K_{15} e^{-\lambda_{5} H} F_{5}\right] \\
= & e^{\bar{m} H}\left[\begin{array}{l}
\bar{K}_{13} e^{-\bar{\lambda}_{3} H} \bar{F}_{3}+ \\
\bar{K}_{14} e^{-\bar{\lambda}_{4} H} \bar{F}_{4}+\bar{K}_{15} e^{-\bar{\lambda}_{5} H} \bar{F}_{5}
\end{array}\right],
\end{aligned}
$$

where

$$
\mathrm{K}_{\mathrm{j}-2}=\lambda_{\mathrm{j}}\left(\delta_{\mathrm{j}}-\lambda_{\mathrm{j}}^{2}+l^{2}\right),
$$




$$
\begin{aligned}
& \bar{K}_{j-2}=\bar{\lambda}_{j}\left(\bar{\delta}_{j}-\bar{\lambda}_{j}^{2}+l^{2}\right) \text {, } \\
& \mathrm{K}_{\mathrm{j}+1}=\mathrm{n}_{\mathrm{j}}\left[\left(\lambda_{\mathrm{j}}+2 \mu_{0}\right) \lambda_{\mathrm{j}}{ }^{2}-\lambda_{0} l^{2}\right]+2 \mathrm{i} l \mu_{0} \lambda_{\mathrm{j}}, \\
& \bar{K}_{j+1}=\bar{n}_{j}\left[\left(\bar{\lambda}_{0}+2 \bar{\mu}_{0}\right) \bar{\lambda}_{j}^{2}-\bar{\lambda}_{0} l^{2}\right]+2 i l \bar{\mu}_{0} \bar{\lambda}_{j} \text {, } \\
& \mathrm{K}_{\mathrm{j}+4}=\mathrm{n}_{\mathrm{j}}\left[\left(\lambda_{\mathrm{j}}+2 \mu_{\mathrm{j}}\right) \lambda_{\mathrm{j}}{ }^{2}-\lambda_{0} l^{2}\right]-2 \mathrm{i} l \mu_{0} \lambda_{\mathrm{j}}, \\
& \bar{K}_{j+4}=\bar{n}_{j}\left[\left(\bar{\lambda}_{0}+2 \bar{\mu}_{0}\right) \bar{\lambda}_{j}^{2}-\bar{\lambda}_{0} l^{2}\right]-2 i l \bar{\mu}_{0} \bar{\lambda}_{j} \text {, } \\
& \mathrm{K}_{\mathrm{j}+7}=\mathrm{ib} \mathrm{F}_{0} \delta_{\mathrm{j}}+2 \mathrm{i} l \mu_{0} \mathrm{n}_{\mathrm{j}} \lambda_{\mathrm{j}}-\mu_{0}\left(\lambda_{\mathrm{j}}^{2}+l^{2}\right), \\
& \bar{K}_{j+7}=i b \bar{F}_{0} \bar{\delta}_{j}+2 i l \bar{\mu}_{0} \bar{n}_{j} \bar{\lambda}_{j}-\bar{\mu}_{0}\left(\bar{\lambda}_{j}^{2}+l^{2}\right), \\
& \mathrm{K}^{\mathrm{j}+10}=\mathrm{ibF}_{0} \delta_{\mathrm{j}}-2 \mathrm{i} l \mu_{0} \mathrm{n}_{\mathrm{j}} \lambda_{\mathrm{j}}-\mu_{0}\left(\lambda_{\mathrm{j}}^{2}+l^{2}\right), \\
& \bar{K}_{j+10}=i b \bar{F}_{0} \bar{\delta}_{j}-2 i l \bar{\mu}_{0} \bar{n}_{j} \bar{\lambda}_{j}-\bar{\mu}_{0}\left(\bar{\lambda}_{j}^{2}+l^{2}\right), \\
& \mathrm{K}_{\mathrm{j}+13}=\mathrm{i} l \mathrm{n}_{\mathrm{j}}-\lambda_{\mathrm{j}}, \\
& \mathrm{K}_{\mathrm{j}+16}=\mathrm{i} \ln _{\mathrm{j}}+\lambda_{\mathrm{j}} \\
& \bar{K}_{j+16}=i l \bar{n}_{j}+\bar{\lambda}_{j} \text {. }
\end{aligned}
$$

(58),

Eliminating $\mathrm{E}_{3}, \mathrm{E}_{4}, \mathrm{E}_{5}, \mathrm{~F}_{3}, \mathrm{~F}_{4}, \mathrm{~F}_{5}, \bar{F}_{3}, \bar{F}_{4}, \bar{F}_{5}$ from Eq.

We get $9 \times 9$ determinant, which gives wave-velocity equation,

$$
\text { i.e. }\left|a_{i j}\right|=0 \text {, where } i, j=1,2, \ldots . .9
$$

Equation (60) gives the dispersion equation of Rayleigh waves for a granular non-homogeneous medium under the influence of gravity.

Where,

$$
\begin{gathered}
\mathrm{a}_{11}=K_{1} e^{-\lambda_{3} H, \mathrm{a}_{12}=} K_{2} e^{-\lambda_{4} H}, \\
\mathrm{a}_{13}=K_{3} e^{-\lambda_{5} H, \mathrm{a}_{14}=} K_{1} e^{\lambda_{3} H}, \\
\mathrm{a}_{15}=K_{2} e^{\lambda_{4} H}, \mathrm{a}_{16}=K_{3} e^{\lambda_{5} H}, \\
\mathrm{a}_{17}=\mathrm{a}_{18}=\mathrm{a}_{19}=0, \\
\mathrm{a}_{21}=K_{4} e^{-\lambda_{3} H}, \mathrm{a}_{22}=K_{5} e^{-\lambda_{4} H}, \\
\mathrm{a}_{23}=K_{6} e^{-\lambda_{5} H}, \mathrm{a}_{24}=K_{7} e^{\lambda_{3} H}, \mathrm{a}_{25}=K_{8} e^{\lambda_{4} H}, \\
\mathrm{a}_{26}=K_{9} e^{\lambda_{5} H,} \mathrm{a}_{27}=\mathrm{a}_{28}=\mathrm{a}_{29}=0,
\end{gathered}
$$

$$
\begin{aligned}
& \mathrm{a}_{31}=K_{10} e^{-\lambda_{3} H}, \mathrm{a}_{32}=K_{11} e^{-\lambda_{4} H}, \\
& \mathrm{a}_{33}=K_{12} e^{-\lambda_{5} H}, \mathrm{a}_{34}=K_{13} e^{\lambda_{3} H}, \\
& \mathrm{a}_{35}=K_{14} e^{\lambda_{4} H}, \mathrm{a}_{36}=\mathrm{K}_{15} e^{\lambda_{5} H}, \mathrm{a}_{37}=\mathrm{a}_{38}=\mathrm{a}_{39}=0 \text {, } \\
& \mathrm{a}_{41}=\mathrm{K}_{16} ; \mathrm{a}_{42}=\mathrm{K}_{17}, \mathrm{a}_{43}=\mathrm{K}_{18}, \\
& \mathrm{a}_{44}=\mathrm{K}_{19}, \mathrm{a}_{45}=\mathrm{K}_{20}, \mathrm{a}_{46}=\mathrm{K}_{47} \text {, } \\
& \mathrm{a}_{47}=\bar{K}_{19}, \mathrm{a}_{48}=\bar{K}_{20}, \mathrm{a}_{49}=\bar{K}_{21} \text {, } \\
& \mathrm{a}_{51}=\mathrm{i} l+\mathrm{n}_{3} \lambda_{3}, \mathrm{a}_{52}=\mathrm{i} l+\mathrm{n}_{4} \lambda_{4}, \\
& \mathrm{a}_{53}=\mathrm{i} l+\mathrm{n}_{5} \lambda_{5}, \mathrm{a}_{54}=\mathrm{i} l-\mathrm{n}_{3} \lambda_{3}, \\
& \mathrm{a}_{55}=\mathrm{i} l-\mathrm{n}_{4} \lambda_{4}, \mathrm{a}_{56}=\mathrm{i} l-\mathrm{n}_{5} \lambda_{5}, \\
& \mathrm{a}_{57}=i l-\bar{n}_{3} \bar{\lambda}_{3}, \mathrm{a}_{58}=i l-\bar{n}_{4} \bar{\lambda}_{4}, \\
& \mathrm{a}_{59}={ }_{i l-\bar{n}_{5}} \bar{\lambda}_{5}, \mathrm{a}_{61}=\delta_{3}, \mathrm{a}_{62}=\delta_{4} \text {, } \\
& a_{63}=\delta_{5}, \delta_{64}=\delta_{3}, a_{65}=\delta_{4}, a_{66}=\delta_{5} \text {, } \\
& \mathrm{a}_{67}=\bar{\delta}_{3}, \mathrm{a}_{68}=\bar{\delta}_{4}, \mathrm{a}_{69}=\bar{\delta}_{5}, \\
& \mathrm{a}_{71}=\mathrm{M}_{0} \mathrm{e}^{\mathrm{mH}} \mathrm{K}_{1}, \mathrm{a}_{72}=\mathrm{M}_{0} \mathrm{e}^{\mathrm{mH}} \mathrm{K}_{2} \text {, } \\
& a_{73}=M_{0} e^{m H} K_{3}, a_{74}=-K_{1} M_{0} e^{m H} \text {, } \\
& \mathrm{a}_{75}=-\mathrm{K}_{2} \mathrm{M}_{0} \mathrm{e}^{\mathrm{mH}} \text {, } \\
& \mathrm{a}_{76}=-\mathrm{K}_{3} \mathrm{M}_{0} \mathrm{e}^{\mathrm{mH}}, \mathrm{a}_{77}=-\bar{K}_{1} \bar{M}_{0} e^{\bar{m} H}, \\
& \mathrm{a}_{78}=-\bar{K}_{2} \bar{M}_{0} e^{\bar{m} H}, \mathrm{a}_{79}=-\bar{K}_{3} \bar{M}_{0} e^{\bar{m} H}, \\
& \mathrm{a}_{81}=\mathrm{K}_{4} \mathrm{e}^{\mathrm{mH}}, \mathrm{a}_{82}=\mathrm{K}_{5} \mathrm{e}^{\mathrm{mH}} \text {, } \\
& \mathrm{a}_{83}=\mathrm{K}_{6} \mathrm{e}^{\mathrm{mH}}, \mathrm{a}_{84}=\mathrm{K}_{7} \mathrm{e}^{\mathrm{mH}} \text {, } \\
& \mathrm{a}_{85}=\mathrm{K}_{8} \mathrm{e}^{\mathrm{mH}}, \mathrm{a}_{86}=\mathrm{K}_{9} \mathrm{e}^{\mathrm{mH}} \text {, } \\
& \mathrm{a}_{87}=\bar{K}_{7} e^{\bar{m} H}, \mathrm{a}_{88}=\bar{K}_{8} e^{\bar{m} H}, \\
& \mathrm{a}_{89}=\bar{K}_{9} e^{\bar{m} H}, \\
& \mathrm{a}_{91}=\mathrm{K}_{10} \mathrm{e}^{\mathrm{mH}}, \mathrm{a}_{92}=\mathrm{K}_{11} \mathrm{e}^{\mathrm{mH}} \text {, }
\end{aligned}
$$




$$
\begin{gathered}
\mathrm{a}_{93}=\mathrm{K}_{12} \mathrm{e}^{\mathrm{mH}}, \mathrm{a}_{94}=\mathrm{K}_{13} \mathrm{e}^{\mathrm{mH}}, \\
\mathrm{a}_{95}=\mathrm{K}_{14} \mathrm{e}^{\mathrm{mH}}, \\
\mathrm{a}_{96}=\mathrm{K}_{15} \mathrm{e}^{\mathrm{mH}}, \mathrm{a}_{97}=\bar{K}_{13} e^{\bar{m} H}, \\
\mathrm{a}_{98}=\bar{K}_{14} e^{\bar{m} H}, \mathrm{a}_{99}=\bar{K}_{15} e^{\bar{m} H} .
\end{gathered}
$$

\section{Particular Cases}

Equation (60) in determinant form gives the wave velocity equation of Rayleigh wave in granular non-homogeneous medium under the influence of gravity, clearly from Eq. (60) we find that wave velocity $\mathrm{c}=\frac{b}{l}$ not only depends on gravity, temperature ,magnetic field, initial stress but also on the non-homogeneity of material.

Case-I In the absence of granular rotations, we get

$$
\begin{gathered}
\underset{M \rightarrow 0}{L t} \underset{s \rightarrow 0}{L t} \lambda_{j=\mathrm{t}_{\mathrm{j}}} \\
\stackrel{L t}{M \rightarrow 0} \underset{s \rightarrow 0}{L t} s \delta_{j=v_{\mathrm{j}}}(\mathrm{j}=3,4,5),
\end{gathered}
$$

where

$$
\mathrm{v}_{\mathrm{j}}=-\frac{i}{b}\left[\beta^{2}\left(\mathrm{t}_{\mathrm{j}}^{2}-l^{2}\right)+\mathrm{b}^{2}+\left(\mathrm{i} l \mathrm{~g}+\mathrm{mi} l \gamma^{2}\right) \eta_{\mathrm{j}}+2 \mathrm{~m} \beta^{2} \mathrm{t}_{\mathrm{j}}\right]
$$

and $t_{j}$ are the roots of the equation by using Eq. (33)

$\alpha^{2} \beta^{2} t_{j}^{6}+\left(3 m \alpha^{2} \beta^{2}\right) t_{j}^{5}+b_{1} t_{j}^{4}+b_{2} t_{j}^{3}+b_{3} t_{j}^{2}+b_{4} t_{j}+b_{5}=0$,

where

$$
\begin{gathered}
\mathrm{b}_{1}=-3 \alpha^{2} \beta^{2} l^{2}+2 \mathrm{~m}^{2} \alpha^{2} \beta^{2}+\mathrm{b}^{2}\left(\alpha^{2}+\beta^{2}\right)+2 \mathrm{~m} \beta^{4}, \\
\mathrm{~b}_{2}=-6 \mathrm{~m} \alpha^{2} \beta^{2} l^{2}+\mathrm{mb} \mathrm{b}^{2}\left(\alpha^{2}+\beta^{2}\right)+6 \mathrm{~m}^{2} \beta^{4}+2 \mathrm{~m} \beta^{2} \mathrm{~b}^{2}, \\
\mathrm{~b}_{3}=-l^{2}\left[-2 l^{2} \alpha^{2} \beta^{2}+2 \mathrm{~m} \beta^{4}+\left(\alpha^{2}+\beta^{2}\right) \mathrm{b}^{2}\right]+2 \mathrm{~m}^{2} \beta^{2}(- \\
\left.\alpha^{2} l^{2}+\mathrm{b}^{2}+2 \mathrm{~m} \beta^{2}\right)+\left(\mathrm{b}^{2}-l^{2} \beta^{2}\right)\left(-\alpha^{2} l^{2}+\mathrm{b}^{2}+2 \mathrm{~m} \beta^{2}\right)+\mathrm{i} l(\mathrm{~g}+ \\
\left.\mathrm{m} \gamma^{2}\right)\left(\mathrm{i} l \mathrm{~g}-\mathrm{i} l \mathrm{~m} \beta^{2}\right), \\
\mathrm{b}_{4}=-2 \mathrm{~m} l^{2} \beta^{2}\left(-\alpha^{2} l^{2}+\mathrm{b}^{2}+2 \mathrm{~m} \beta^{2}\right)+\left(\mathrm{mb}-\mathrm{m} l^{2} \beta^{2}\right) \\
\left(-\alpha^{2} l^{2}+\mathrm{b}^{2}+2 \mathrm{~m} \beta^{2}\right)+\mathrm{im} l\left(\mathrm{~g}+\mathrm{m} \gamma^{2}\right)\left(\mathrm{i} l \mathrm{~g}-\mathrm{i} l \mathrm{~m} \beta^{2}\right), \\
\mathrm{b}_{5}=-l^{2}\left[\left(\mathrm{~b}^{2}-l^{2} \beta^{2}\right)\left(-\alpha^{2} l^{2}+\mathrm{b}^{2}+2 \mathrm{~m} \beta^{2}\right)+\mathrm{i} l\left(\mathrm{~g}+\mathrm{m} \gamma^{2}\right)(\mathrm{i} l \mathrm{~g}\right. \\
\left.-\mathrm{i} l \mathrm{~m} \beta^{2}\right) .
\end{gathered}
$$

So Eq. (60) together with relation given by Eq. (62) forms the dispersion equation for the semi-infinite elastic, isotropic and non-homogeneous medium overlain by a granular layer under the influence of gravity, magnetic field and temperature.

Case-II In the absence of non-homogeneity, Eq. (60) gives the Dispersion equation of Rayleigh waves for a granular medium under the influence of gravity, magnetic field and temperature.

Where,

$$
\begin{gathered}
\mathrm{a}_{71}=\mathrm{M}_{0} \mathrm{~K}_{1}, \mathrm{a}_{72}=\mathrm{M}_{0} \mathrm{~K}_{2}, \mathrm{a}_{73}=\mathrm{M}_{0} \mathrm{~K}_{3}, \\
\mathrm{a}_{74}=-\mathrm{M}_{0} \mathrm{~K}_{1}, \mathrm{a}_{75}=-\mathrm{M}_{0} \mathrm{~K}_{2}, \\
\mathrm{a}_{76}=-\mathrm{M}_{0} \mathrm{~K}_{3}, \mathrm{a}_{77}=-\bar{K}_{1} \bar{M}_{0}, \\
\mathrm{a}_{78}=-\bar{K}_{2} \bar{M}_{0}, \mathrm{a}_{79}=-\bar{K}_{3} \bar{M}_{0}, \\
\mathrm{a}_{81}=\mathrm{K}_{4}, \mathrm{a}_{82}=\mathrm{K}_{5}, \mathrm{a}_{83}=\mathrm{K}_{6}, \\
\mathrm{~K}_{7}, \mathrm{a}_{85}=\mathrm{K}_{8}, \mathrm{~K}_{86}=\mathrm{K}_{9}, \mathrm{a}_{87}=\bar{K}_{7}, \\
\mathrm{a}_{88}=\bar{K}_{8}, \mathrm{a}_{89}=\bar{K}_{9}, \\
\mathrm{a}_{91}=\mathrm{K}_{10}, \mathrm{a}_{92}=\mathrm{K}_{11}, \mathrm{a}_{93}=\mathrm{K}_{12}, \\
\mathrm{a}_{94}=\mathrm{K}_{13}, \mathrm{a}_{95}=\mathrm{K}_{14}, \mathrm{a}_{96}=\mathrm{K}_{15}, \\
\mathrm{a}_{97}=\bar{K}_{13}, \mathrm{a}_{98}=\bar{K}_{14}, \mathrm{a}_{99}=\bar{K}_{15},
\end{gathered}
$$

and rest of $\mathrm{a}_{\mathrm{ij}}$ 's are same as in Eq. (61).

Case-III In the absence of granular rotations and nonhomogeneity, we get

$$
\begin{gathered}
\stackrel{L t}{m \rightarrow 0} \underset{M \rightarrow 0}{\operatorname{Lt}} \underset{s \rightarrow 0}{\operatorname{Lt}} \lambda_{j}=\mathrm{x}_{\mathrm{j}}, \\
\underset{m \rightarrow 0}{L t} \stackrel{L t}{M \rightarrow 0} \underset{s \rightarrow 0}{L t}\left(s \delta_{j}\right)=\mathrm{W}_{\mathrm{j}}(\mathrm{j}=3,4,5)
\end{gathered}
$$

where

$$
\begin{gathered}
\mathrm{W}_{\mathrm{j}}=-\frac{i}{b}\left[\beta^{2}\left(x_{j}^{2}-l^{2}\right)+b^{2}+i l g n_{j}\right], \\
\eta_{\mathrm{j}}=\frac{i l g}{\alpha^{2} x_{j}{ }^{2}-\alpha^{2} l^{2}+b^{2}}
\end{gathered}
$$

and $\mathrm{x}_{\mathrm{j}}$ are the roots of the equation

$$
\begin{gathered}
\alpha^{2} \beta^{2} \mathrm{x}_{\mathrm{j}}^{6}+\left[\left(\alpha^{2}+\beta^{2}\right) \mathrm{b}^{2}-3 \alpha^{2} \beta^{2} l^{2}\right] \mathrm{x}_{\mathrm{j}}^{4}+\left[2 l^{4} \alpha^{2} \beta^{2}-\mathrm{b}^{2} l^{2}\right. \\
\left(\alpha^{2}+\beta^{2}\right) \\
\left.+\left(\mathrm{b}^{2}-l^{2} \beta^{2}\right)\left(\mathrm{b}^{2}-l^{2} \alpha^{2}\right)-l^{2} \mathrm{~g}^{2}\right] \mathrm{x}_{\mathrm{j}}^{2}+\left[( \mathrm { b } ^ { 2 } - l ^ { 2 } \beta ^ { 2 } ) l ^ { 2 } \left(\alpha^{2} l^{2}-\right.\right. \\
\left.\left.\mathrm{b}^{2}\right)+l^{4} \mathrm{~g}^{2}\right]=0
\end{gathered}
$$

Thus the equation $\left|a_{i j}\right|=0$, where $i, j=1,2, \ldots .9$ where $a_{i j}$ 's are given by Eq. (63) gives the Dispersion eq- 
uation for the semi-infinite, elastic and isotropic medium overlain by a granular layer under the influence of gravity.

Case-IV In the absence of gravity, magnetic field $\mathrm{H}_{0}=0$, temperature $\mathrm{T}_{0}=0$, initial stress $\mathrm{P}_{0}=0$ and non-homogeneity, we get

$$
\begin{gathered}
\lambda_{5}{ }^{2}=l^{2}-\frac{b^{2}}{\alpha^{2}}, \\
2 l^{2} \beta^{2}-b^{2}-i b \beta^{2} s^{\prime}- \\
\left(\lambda_{3}^{2}, \lambda_{4}{ }^{2}\right)=\frac{2 i b l^{2} s \pm b \sqrt{\left(b-i \beta^{2} s^{\prime}\right)^{2}-4 b^{2} s s^{\prime}}}{2\left(\beta^{2}-i b s\right)}
\end{gathered}
$$
to

so by making $\eta_{3}, \eta_{4} \rightarrow 0$, the dispersion Eq. (60) reduces

$$
\left|b_{i j}\right|=0 \text { where } i, j=1,2, \ldots . .9 \text {, }
$$

where

$$
\begin{aligned}
& \mathrm{b}_{11}=r_{1} e^{-\lambda_{3} H}, \mathrm{~b}_{12}=-r_{1} e^{\lambda_{3} H}, \\
& \mathrm{~b}_{13}=r_{2} e^{-\lambda_{4} H}, \mathrm{~b}_{14}=-r_{2} e^{\lambda_{4} H}, \\
& \mathrm{~b}_{15}=\mathrm{b}_{16}=\mathrm{b}_{17}=\mathrm{b}_{18}=\mathrm{b}_{19}=0 \text {, } \\
& \mathrm{b}_{21}=r_{3} e^{-\lambda_{3} H}, \mathrm{~b}_{22}=-r_{3} e^{\lambda_{3} H}, \\
& \mathrm{~b}_{23}=r_{4} e^{-\lambda_{4} H}, \mathrm{~b}_{24}=-r_{4} e^{\lambda_{4} H}, \mathrm{~b}_{25}=r_{5} e^{-\lambda_{5} H}, \\
& \mathrm{~b}_{26}=-r_{5} e^{\lambda_{5} H}, \mathrm{~b}_{27}=\mathrm{b}_{28}=\mathrm{b}_{29}=0 \text {, } \\
& \mathrm{b}_{31}=r_{6} e^{-\lambda_{3} H}, \mathrm{~b}_{32}=-r_{6} e^{\lambda_{3} H}, \\
& \mathrm{~b}_{33}=r_{7} e^{-\lambda_{4} H}, \mathrm{~b}_{34}=-r_{7} e^{\lambda_{4} H}, \mathrm{~b}_{35}=r_{8} e^{-\lambda_{5} H} \text {, } \\
& \mathrm{b}_{36}=-r_{8} e^{\lambda_{5} H}, \mathrm{~b}_{37}=\mathrm{b}_{38}=\mathrm{b}_{39}=0, \\
& \mathrm{~b}_{41}=-\lambda_{3}, \mathrm{~b}_{42}=\lambda_{3}, \mathrm{~b}_{43}=-\lambda_{4} \text {, } \\
& \mathrm{b}_{44}=\lambda_{4}, \mathrm{~b}_{45}=\mathrm{i} l \text {, } \\
& \mathrm{b}_{46}=\mathrm{i} l, \mathrm{~b}_{47}=\bar{\lambda}_{3}, \mathrm{~b}_{48}=\bar{\lambda}_{4}, \mathrm{~b}_{49}=\mathrm{i} l \text {, } \\
& \mathrm{b}_{51}=\mathrm{b}_{52}=\mathrm{b}_{53}=\mathrm{b}_{54}=\mathrm{i} l, \mathrm{~b}_{55}=\lambda_{5} \text {, } \\
& \mathrm{b}_{56}=-\lambda_{5}, \mathrm{~b}_{57}=\mathrm{b}_{58}=\mathrm{i} l, \mathrm{~b}_{59}=-\bar{\lambda}_{5} \text {, } \\
& \mathrm{b}_{61}=\mathrm{b}_{62}=\delta_{3}, \mathrm{~b}_{63}=\mathrm{b}_{64}=\delta_{4}, \\
& \mathrm{~b}_{65}=\mathrm{b}_{66}=0, \mathrm{~b}_{67}=\bar{\delta}_{3}, \mathrm{~b}_{68}=\bar{\delta}_{4}, \mathrm{~b}_{69}=0 \text {, }
\end{aligned}
$$

$$
\begin{gathered}
\mathrm{b}_{71}=\mathrm{M}_{0} \mathrm{r}_{1}, \mathrm{~b}_{72}=-\mathrm{M}_{0} \mathrm{r}_{1}, \\
\mathrm{~b}_{73}=\mathrm{M}_{0} \mathrm{r}_{2}, \mathrm{~b}_{74}=-\mathrm{M}_{0} \mathrm{r}_{2}, \mathrm{~b}_{75}=\mathrm{b}_{76}=0, \\
\mathrm{~b}_{77}=\bar{M}_{0} \bar{r}_{1}, \mathrm{~b}_{78}=\bar{M}_{0} \bar{r}_{2}, \mathrm{~b}_{79}=0,(66) \\
\mathrm{b}_{81}=\mathrm{r}_{3}, \mathrm{~b}_{82}=-\mathrm{r}_{3}, \mathrm{~b}_{83}=\mathrm{r}_{4}, \\
\mathrm{~b}_{84}=-\mathrm{r}_{4}, \mathrm{~b}_{85}=\mathrm{r}_{5}=\mathrm{b}_{86}, \mathrm{~b}_{87}=-\bar{r}_{3}, \mathrm{~b}_{88}=-\bar{r}_{4}, \\
\mathrm{~b}_{95}=\mathrm{r}_{8}, \mathrm{~b}_{96}=-\mathrm{r}_{81}, \mathrm{~b}_{97}=\mathrm{r}_{6}=\mathrm{b}_{92}, \mathrm{~b}_{93}=\mathrm{b}_{94}=\mathrm{r}_{7}, \\
\mathrm{~b}_{99}=-\bar{r}_{98} .
\end{gathered}
$$

and

$$
\begin{aligned}
& \mathrm{r}_{1}=\lambda_{3}\left(\delta_{3}-\lambda_{3}{ }^{2}+l^{2}\right), \bar{r}_{1}=\bar{\lambda}_{3}\left(\bar{\delta}_{3}-\bar{\lambda}_{3}^{2}+l^{2}\right), \\
& \mathrm{r}_{2}=\lambda_{4}\left(\delta_{4}-\lambda_{4}^{2}+l^{2}\right), \bar{r}_{2}=\bar{\lambda}_{4}\left(\bar{\delta}_{4}-\bar{\lambda}_{4}^{2}+l^{2}\right), \\
& \mathrm{r}_{3}=2 \mathrm{i} l \mu_{0} \lambda_{3}, \bar{r}_{3}=2 i l \bar{\mu}_{0} \bar{\lambda}_{3}, \\
& \mathrm{r}_{4}=2 \mathrm{i} l \mu_{0} \lambda_{4}, \bar{r}_{4}=2 i l \bar{\mu}_{0} \bar{\lambda}_{4} \\
& { }^{\mathrm{r}_{5}=} \mu_{0}\left(2 l^{2}-\frac{b^{2}}{\beta^{2}}\right)^{,} \overline{r_{5}}=\mu_{0}\left(2 l^{2}-\frac{b^{2}}{\beta^{2}}\right) \\
& \mathrm{r}_{6}=\mathrm{ib} \mathrm{F} \delta_{0}-\mu_{0}\left(\lambda_{3}^{2}+l^{2}\right), \bar{r}_{6}=\mathrm{ib} \\
& \bar{F}_{0} \bar{\delta}_{3}-\bar{\mu}_{0}\left(\bar{\lambda}_{3}^{2}+l^{2}\right) \\
& \mathrm{r}_{7}=\mathrm{ib} \mathrm{F}{ }_{0} \delta_{4}-\mu_{0}\left(\lambda_{4}^{2}+l^{2}\right), \bar{r}_{7}=\mathrm{ib} \\
& \bar{F}_{0} \bar{\delta}_{4}-\bar{\mu}_{0}\left(\bar{\lambda}_{4}^{2}+l^{2}\right), \\
& \mathrm{r}_{8}=2 \mathrm{i} l \mu_{0} \lambda_{5}, \bar{r}_{8}=2 i l \bar{\mu}_{0} \bar{\lambda}_{5}
\end{aligned}
$$

Eq. (67) gives the Dispersion equation of Rayleigh waves for a granular medium in the absence of gravity and non-homogeneity and is in complete agreement with that obtained by Bhattacharaya et al. [22].

Case-V In the absence of gravity, granular rotations, magnetic field $\mathrm{H}_{0}=0$, temperature $\mathrm{T}_{0}=0$, initial stress $\mathrm{P}_{0}=0$ and non-homogeneity.

Now using Eq. (65) and (66) into Eq. (67), we get 


$$
\begin{aligned}
& \underset{m \rightarrow 0}{L t} \stackrel{L t}{M \rightarrow 0 t} \underset{s \rightarrow 0}{L t}\left(\lambda_{3}^{2}, \lambda_{4}^{2}\right)=\left(l^{2}, l^{2}-\frac{b^{2}}{\beta^{2}}\right) \\
& \underset{m \rightarrow 0}{L t} \stackrel{L t}{M \rightarrow 0} \underset{s \rightarrow 0}{L t}\left(s \delta_{3}\right)=-\mathrm{ib} \\
& \underset{m \rightarrow 0}{L t} \stackrel{L t}{M \rightarrow 0} \underset{s \rightarrow 0}{L t}\left(s \delta_{4}\right)=0 \\
& \underset{m \rightarrow 0}{L t} \underset{M \rightarrow 0}{L t} \underset{s \rightarrow 0}{L t} \delta_{4}=-\frac{b^{2}}{\beta^{2}} \\
& \underset{m \rightarrow 0}{L t} \stackrel{L t}{M \rightarrow 0} \underset{s \rightarrow 0}{L t} r_{6}=-\mu\left(2 l^{2}-\frac{b^{2}}{\beta^{2}}\right) \\
& \underset{m \rightarrow 0}{L t} \underset{M \rightarrow 0}{L t} \underset{s \rightarrow 0}{L t} r_{7}=-\mu\left(2 l^{2}-\frac{b^{2}}{\beta^{2}}\right)
\end{aligned}
$$

Similar results are also holds for lower medium.

Now using Eq. (68) into Eq. (67), then after some simplification we get

$6 \times 6$ determinantal equation

$$
\left|\mathrm{d}_{\mathrm{ij}}\right| \quad=0 \text { where } \mathrm{i}, \mathrm{j}=1,2,3, \ldots .6,
$$

where

$$
\begin{gathered}
\mathrm{d}_{11}=2 l \lambda_{4} e^{\lambda_{4} H}, \mathrm{~d}_{12}=\left(2 l^{2}-\frac{b^{2}}{\beta^{2}}\right) e^{\lambda_{5} H}, \\
\mathrm{~d}_{13}=-2 l \lambda_{4} e^{-\lambda_{4} H}, \\
\mathrm{~d}_{14}=\left(2 l^{2}-\frac{b^{2}}{\beta^{2}}\right) e^{-\lambda_{5} H}, \mathrm{~d}_{15}=\mathrm{d}_{16}=0, \\
\mathrm{~d}_{21}=\left(2 l^{2}-\frac{b^{2}}{\beta^{2}}\right) e^{\lambda_{4} H}, \mathrm{~d}_{22}=2 l \lambda_{5} e^{\lambda_{5} H}, \\
\mathrm{~d}_{23}=\left(2 l^{2}-\frac{b^{2}}{\beta^{2}}\right) e^{-\lambda_{4} H}, \mathrm{~d}_{24}=-2 l \lambda_{5} e^{-\lambda_{5} H}, \\
\mathrm{~d}_{25}=\mathrm{d}_{26}=0, \\
\mathrm{~d}_{31}=-\lambda_{4}, \mathrm{~d}_{32}=-l, \mathrm{~d}_{33}=\lambda 4, \mathrm{~d}_{34}=-l, \\
\mathrm{~d}_{35}=\bar{\lambda}_{4}, \mathrm{~d}_{36}=l, \\
\mathrm{~d}_{41}=-l, \mathrm{~d}_{42}=-\lambda_{5}, \mathrm{~d}_{43}=-l, \mathrm{~d}_{44}=\lambda_{5},
\end{gathered}
$$

$$
\begin{gathered}
\mathrm{d}_{45}=l, \mathrm{~d}_{46}=\bar{\lambda}_{5}, \\
\mathrm{~d}^{2} 1=2 l^{2}-\frac{b^{2}}{\beta^{2}}, \mathrm{~d}_{52}=2 l \lambda_{5}, \\
\mathrm{~d}_{53}=2 l^{2}-\frac{b^{2}}{\beta^{2}}, \mathrm{~d}_{54}=-2 l \lambda_{5}, \\
\mathrm{~d}_{55}=-\frac{\bar{\mu}_{0}}{\mu_{0}}\left(2 l^{2}-\frac{b^{2}}{\beta^{2}}\right), \mathrm{d}_{56}=21 \frac{\bar{\mu}_{0}}{\mu_{0}} \bar{\lambda}_{5} \\
\mathrm{~d}_{61}=2 l \lambda_{4}, \mathrm{~d}_{62}=2 l^{2}-\frac{b^{2}}{\beta^{2}}, \mathrm{~d}_{63}=-2 l \lambda_{4}, \\
\mathrm{~d}_{64}=2 l^{2}-\frac{b^{2}}{\beta^{2}}, \mathrm{~d}_{65}=-2 l \frac{\bar{\mu}_{0}}{\mu_{0}} \bar{\lambda}_{4}, \\
\mathrm{~d}_{66}=-\frac{\bar{\mu}_{0}}{\mu_{0}}\left(2 l^{2}-\frac{b^{2}}{\beta^{2}}\right)
\end{gathered}
$$

Thus Eq. (69) gives the Dispersion equation of Rayleigh waves for semi-infinite elastic and isotropic medium overlain by granular layer of thickness $\mathrm{H}$ in the absence of gravity and non-homogeneity is in complete agreement with the equation obtained by Ewing et al. [22]

\section{Numerical Analysis}

Numerical results have been obtained graphically to show the effect non-homogeneities and phase velocity on initial stress and dimensionless wave number. The parameters for the material are as

$$
\begin{gathered}
\mathrm{C}_{11}=135 \mathrm{GPa}, \mathrm{C}_{13}=67.9 \mathrm{GPa}, \\
\mathrm{C}_{44}=22.2 \mathrm{GPa}, \mathrm{C}_{33}=113 \mathrm{GPa}, \\
\rho_{0}=7.5 \times 10^{3} \mathrm{Kg} / \mathrm{m}^{3} .
\end{gathered}
$$

Various graphs are plotted with the help of MathCAD.

Fig. 2 shows the effect of initial compression on the Rayleigh Waves, it is obvious that Rayleigh wave velocity decreases with an increasing of the various values of the initial stress $\mathrm{P}$ also with the wave number.

Fig. 3 represents the variation of phase velocity with dimensionless less wave number at different values of initial stress. The three modes of Rayleigh waves have been plotted at two different values of initial stress i.e. at $\mathrm{P}=1$ and $\mathrm{P}$ $=0.1$. The value of magnetic field and temperature is fixed at 0.4 Tesla and $293 \mathrm{~K}$. It is clear from fig. 3 as the value of initial compression increases the phase velocity decreases sharply with dimension less wave number.

Fig. 4 is plotted to observe the effect of various non- 
homogeneities factor $W^{\prime}$ in (\%) on Rayleigh waves velocity with respect wave number at $\mathrm{P}=1$ and $\mathrm{P}=0.1$. In graph

$W$ represents the zero ${ }^{\text {th }}$ level of non-homogeneities.

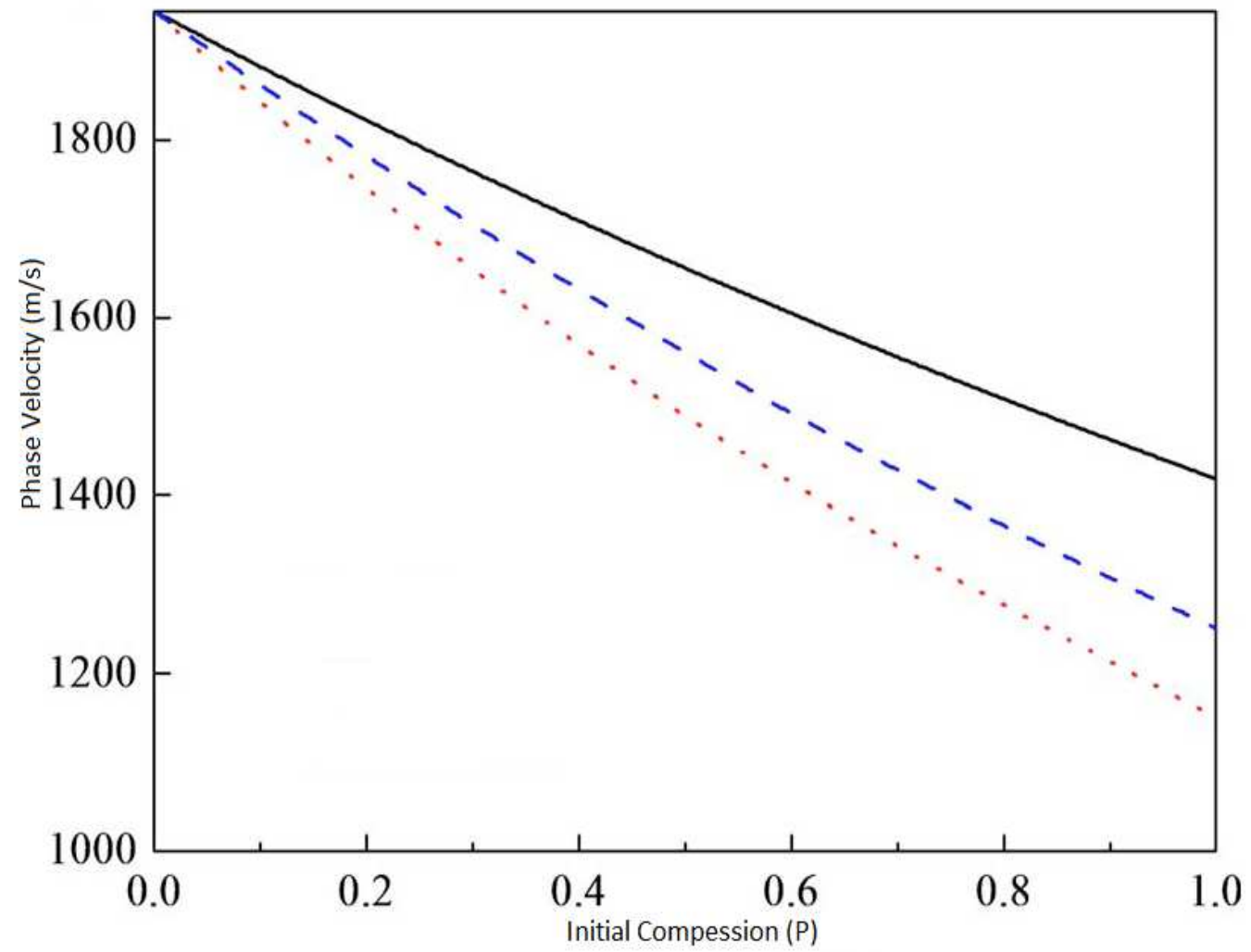

Figure 2. Variation of Rayleigh waves velocity respect to initial stress with the various values of the wave number, $H=0.4$ Tesla, $g=9.8 \mathrm{~m} / \mathrm{s}, T=293 \mathrm{~K}$, granular rotations $=0$.

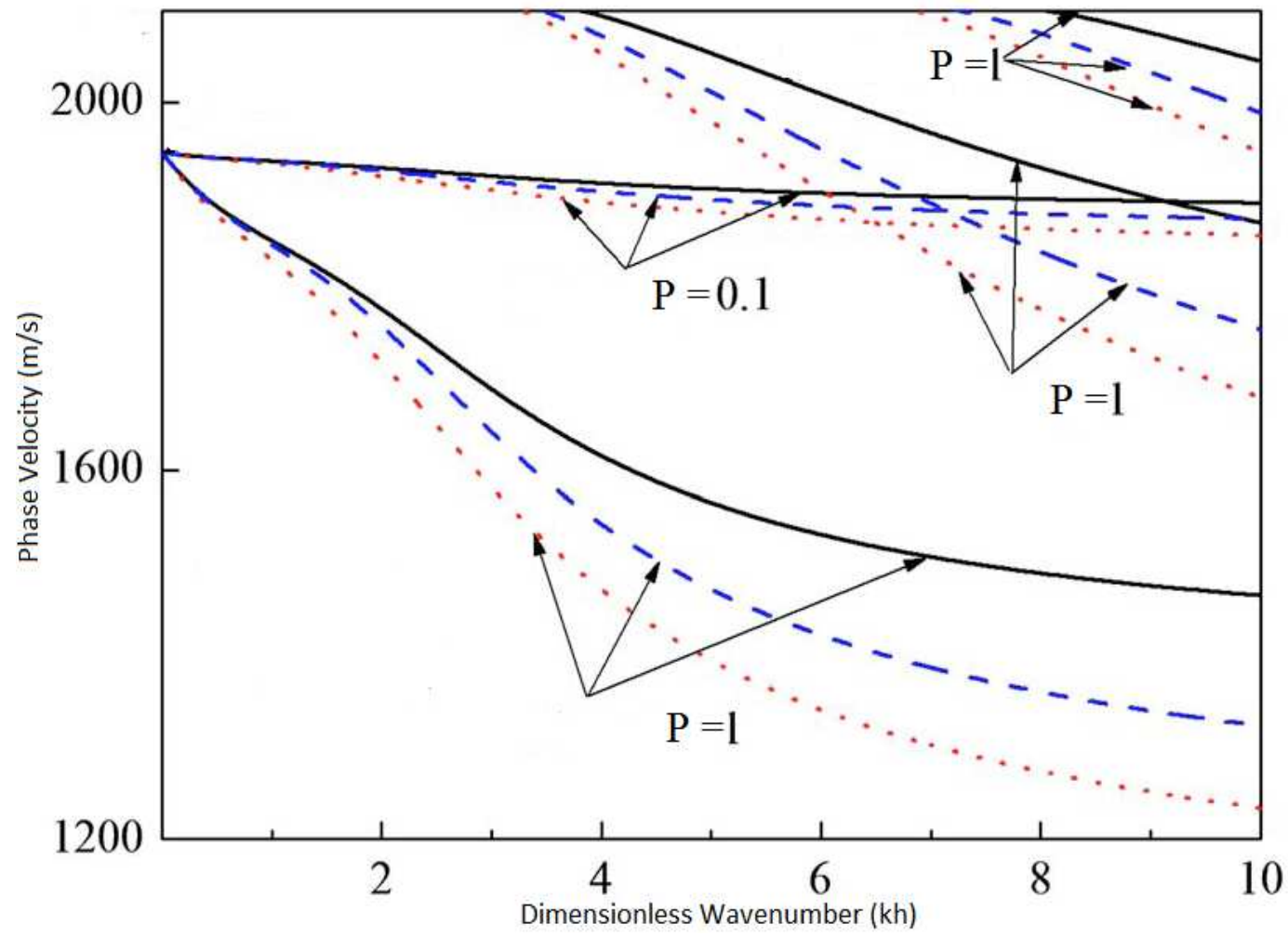

Figure 3. Variation of Rayleigh waves velocity respect wave number, $H=0.4$ Tesla, $T=293 \mathrm{~K}, g=9.8 \mathrm{~m} / \mathrm{s}, P=1, P=0.1$, granular rotations $=0$. 


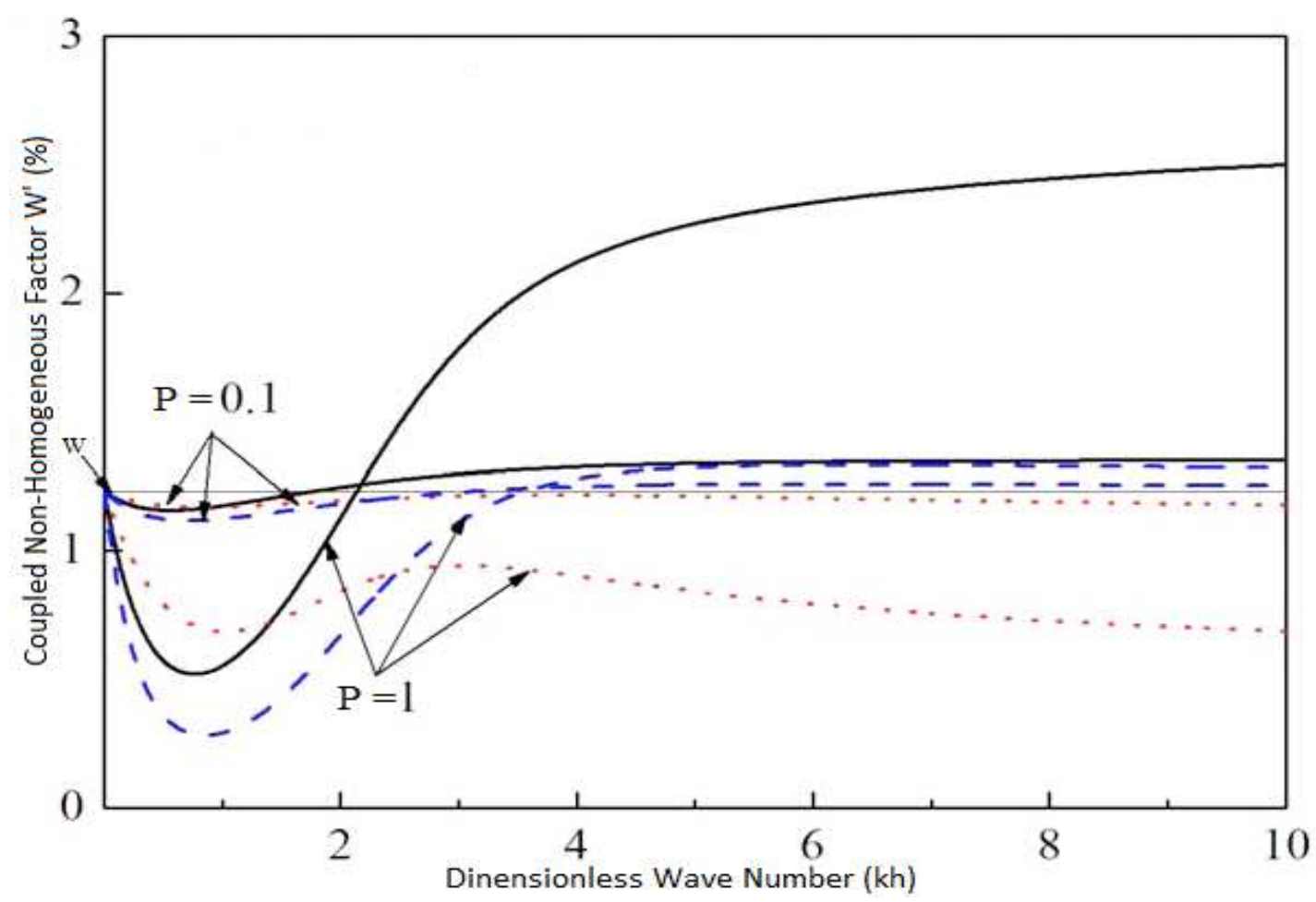

Figure 4. Effect of various non-homogeneities in (\%) on Rayleigh waves velocity with respect wave number keeping initial stress at $P=1$ and $P=0.1$.

\section{Conclusions}

The frequency equation contains terms involving gravity and non-homogeneity, so the phase velocity not only depends on gravity field but also on the non-homogeneity of the material medium, magnetic field, temperature, initial stress and granular notations. The transcendental equation (60) in determinant form gives the wave velocity equation of Rayleigh waves in magneto, thermo, granular nonhomogeneous medium under the influence of gravity and initial compression. Clearly, from equation (60) we find that wave velocity not only depends on gravity but also on the non-homogeneity of material medium, temperature, magnetic field, friction and initial stress. The results are in complete agreement with the corresponding classical results in the absence of all factors. Further in the absence of gravity, temperature, non-homogeneity, magnetic field, initial stress, equation (65) gives the Dispersion equation of Rayleigh waves for a granular medium and it is same as obtained by Bhattacharaya et. al [22].

Also, equation (69) gives the Dispersion equation of Rayleigh waves for semi infinite elastic and isotropic medium overlain by granular layer of thickness $\mathrm{H}$ in the absence of gravity, temperature, granular rotations, nonhomogeneity, magnetic field, initial compression and is incomplete agreement with the equation as obtained by Ewing et al [23].

The exact solution for inhomogeneous half-space subjected to gravity field, temperature field and mechanical field is obtained. All material coefficients are assumed to have the same exponent-law dependence on the depth of the half space. The governing equations in Cartesian coordinates are recorded for future reference. The present study reveals the influence of magnetic field, temperature, gravity and non-homogeneity on the propagation of Rayleigh wave type in a granular half space supporting an initially stressed granular layer. The dispersion equation in the form of ninth order determinant form has been derived. The real part gives the velocity of Rayleigh waves and its imaginary part determines the attenuation of the waves due to granular nature of the medium.

\section{Acknowledgements}

The authors are also thankful to the referees for their valuable comments.

\section{References}

[1] Landau L. D., E. M. Lifshitz, Theory of Elasticity, 3rd ed. Pergamon, New York, 1986.

[2] Datta B.K., Some observation on interactions of Rayleigh waves in an elastic solid medium with the gravity field, Rev. Roumaine Sci. Tech. Ser. Mec. Appl. 31, pp. 369-374, 1986.

[3] Abd-Alla A. M., Ahmed S. M., Propagation of Love waves in a non-homogeneous orthotropic elastic layer under initial stress overlying semi-infinite medium, Applied Mathematics and Computation 106, pp. 265-275, 1999.

[4] El-Naggar A. M., On the dynamical problem of a generalized thermoelastic granular infinite cylinder under initial stress," Astrophysics and Space Science 190, pp. 177-190, 1992. 
[5] Ahmed S. M., Influence of gravity on the propagation of waves in granular medium. Appl.Math.Compu.101, 2-3, pp. 269-280, 1999.

[6] Sharma J. N., Kaur D., Rayleigh waves in rotating thermoelastic solids with voids. Int. J. of appl. Math. and Mech., 6(3), pp.43-61, 2010.

[7] Oshima, N., Asymmetrical stress tensor and its application to a granular medium, in Proceedings of the 3rd Japan National Congress for Applied Mechanics, 77, pp. 77-83, $1955 \mathrm{a}$.

[8] Oshima, N., Men. Unifying study of basic problems in engineering science by means of geometry, Kondo, K. (ed.), 1-3, Gakujutsu Bunken Fukyo-Kai, Tokyo, $1955 b$.

[9] Paria, G., Bending of a shallow spherical shell under uniform pressures with the boundary partly clamped and partly simply-supported, Bulletin of the Calcutta Mathematical Society $52,79-86,1960$.

[10] Ahmed S.M., Abo-Dahab S. M., Propagation of Love Waves in an Orthotropic Granular Layer Under Initial Stress Overlying Semi-infinite Granular Medium, Journal of Vibration and Control, 2010. DOI: 10.1177/1077546309341154.

[11] Abd-Alla A. M., Hammad H. A. H., Abo-Dahab S. M., Rayleigh waves in a magnetoelastic half-space of orthotropic material under influence of initial stress and gravity field, Applied Mathematics and Computation,154, pp. 583-597, 2000.

[12] Willson W., The propagation of magneto-thermo, elastic plane waves, Proc.Cambridge Philos. Soc., 59. pp. 483-488, 1963.

[13] Yu C. P., Tang S., Magneto elastic waves in initially stressed conductors. Z. Angew. Math. Phys.17. pp. 766-775, 1966.

[14] Gupta K.C., Sethi M., Kakar R., Propagation of Surface Waves in Non-Homogeneous Granular Medium Under the Influence of Gravity, International Journal of Computational and Applied Mathematics , 5(3), pp. 341-357, 2010.

[15] Xianhai Song, Li Tang, Xiaochun Lv , Hongping Fang , Hanming Gu, Application of particle swarm optimization to interpret Rayleigh wave dispersion curves, Journal of Applied Geophysics, http://dx.doi.org/10.1016/j.jappgeo.2012.05.011.

[16] Kakar R., Kakar S., Propagation of Rayleigh Waves in a Non-Homogeneous Granular Media, Journal of Chemical, Biological and Physical Sciences, 3(1), pp. 464-478, 20122013.

[17] Kakar R., Kakar S., Effect of Initial Stress and Gravity on Rayleigh Waves Propagating in Non-Homogeneous Orthotropic Elastic Media, International Journal of Applied Engineering and Technology, 2 (4), pp. 9-16, 2012.

[18] Kakar R., Kakar S., Propagation of Rayleigh waves in nonhomogeneous orthotropic elastic media under the influence of gravity, compression, rotation and magnetic field, J. Chem. Bio. Phy. Sci., Sec. C., 3(1), pp. 801-819, 2012-2013.

[19] Kakar R., Kakar S., Influence of gravity and temperature on Rayleigh waves in non-homogeneous, general viscoelastic media of higher order, International Journal of Physical and Mathematical Sciences, 4(1), pp. 62-70, 2013.

[20] Britan A., Ben-Dor G., Shock tube study of the dynamical behavior of granular materials, International Journal of Multiphase Flow 32, pp. 623-642, 2006.

[21] Danoyan Z. N., Piliposian G. T., Surface electro-elastic Love waves in a layered structure with a piezoelectric substrate and a dielectric layer, International Journal of Solids and Structures 44(18-19), pp. 5829-5847, 2007.

[22] Du, J., Xiaoying, J., Wang, J. and Xian, K., 2007, Love wave propagation in functionally graded piezoelectric material layer, Ultrasonics 46(1), 13-22.

[23] Eskandari, M. and Shodja, H. M., Love waves propagation in functionally graded piezoelectric materials with quadratic variation, Journal of Sound and Vibration 313(1-2), 195204, 2008.

[24] Du, J., Xian, K., Wang, J. and Yong, Y., Love wave propagation in piezoelectric layered structure with dissipation, Ultrasonics 49(2), 281-286, 2009.

[25] Bhattacharyya R. K., Rayleigh waves in granular medium. Pure Appl. Geophys.62, 3, pp. 13-22, 1965.

[26] Ewing W. M., Jardetzky W. S., Press F., Elastic waves in layered media, Mcgraw-Hill,New York, 1957. 